\title{
EVOLUTION OF MOLECULAR AND ATOMIC GAS PHASES IN THE MILKY WAY
}

\author{
Jin Koda ${ }^{1,2}$, Nick Scoville ${ }^{2}$, AND Mark Heyer ${ }^{3}$ \\ ${ }^{1}$ Department of Physics and Astronomy, Stony Brook University, Stony Brook, NY 11794-3800, USA; jin.koda@stonybrook.edu \\ ${ }^{2}$ California Institute of Technology, MC 249-17, 1200 East California Boulevard, Pasadena, CA 91125, USA \\ ${ }^{3}$ Department of Astronomy, University of Massachusetts, Amherst, MA 01003, USA \\ Received 2015 October 9; accepted 2016 March 18; published 2016 May 24
}

\begin{abstract}
We analyze radial and azimuthal variations of the phase balance between the molecular and atomic interstellar medium (ISM) in the Milky Way (MW) using archival $\mathrm{CO}(J=1-0)$ and $\mathrm{HI} 21 \mathrm{~cm}$ data. In particular, the azimuthal variations - between the spiral arm and interarm regions - are analyzed without any explicit definition of the spiral arm locations. We show that the molecular gas mass fraction, i.e., $f_{\mathrm{mol}}=\Sigma_{\mathrm{H}_{2}} /\left(\Sigma_{\mathrm{HI}}+\Sigma_{\mathrm{H}_{2}}\right)$, varies predominantly in the radial direction: starting from $\sim 100 \%$ at the center, remaining $\gtrsim 50 \%$ to $R \sim 6 \mathrm{kpc}$ and decreasing to $\sim 10 \%-20 \%$ at $R=8.5 \mathrm{kpc}$ when averaged over the whole disk thickness (from $\sim 100 \%$ to $\gtrsim 60 \%$, then to $\sim 50 \%$ in the midplane). Azimuthal, arm-interarm variations are secondary: only $\sim 20 \%$ in the globally molecule-dominated inner MW, but becoming larger, $40 \%-50 \%$, in the atom-dominated outskirts. This suggests that in the inner MW the gas remains highly molecular $\left(f_{\text {mol }}>50 \%\right)$ as it moves from an interarm region into a spiral arm and back into the next interarm region. Stellar feedback does not dissociate molecules much, and the coagulation and fragmentation of molecular clouds dominate the evolution of the ISM at these radii. The trend differs in the outskirts where the gas phase is globally atomic $\left(f_{\text {mol }}<50 \%\right)$. The $\mathrm{HI}$ and $\mathrm{H}_{2}$ phases cycle through spiral arm passage there. These different regimes of ISM evolution are also seen in external galaxies (e.g., the LMC, M33, and M51). We explain the radial gradient of $f_{\text {mol }}$ using a simple flow continuity model. The effects of spiral arms on this analysis are illustrated in the Appendix.
\end{abstract}

Key words: evolution - Galaxy: disk - Galaxy: evolution - ISM: atoms - ISM: clouds - ISM: molecules

\section{INTRODUCTION}

The evolution of the molecular and atomic gas phases in the Milky Way (MW) has been an issue of debate for a long time (e.g., Scoville \& Hersh 1979; Blitz \& Shu 1980; see Heyer \& Dame 2015 for review). In fact, two contradictory scenarios have been suggested for the evolution of gas across spiral arms. The classic scenario posits a rapid phase transition from interarm HI gas to molecular clouds (MCs) ${ }^{4}$ in spiral arms, and back into the ionized and atomic phases by photodissociation associated with star formation in the spiral arms (e.g., Blitz \& Shu 1980; Cohen et al. 1980). Alternatively, the dynamically driven scenario involves little phase transition and suggests that the evolution is driven by the coagulation and fragmentation of MCs around spiral arms, assembling pre-existing small interarm MCs into more massive ones in spiral arms and shearing them into smaller MCs toward the next interarm region (e.g., Scoville \& Hersh 1979; Vogel et al. 1988). The difference between the two scenarios (i.e., diffuse HI versus dense $\mathrm{H}_{2}$ in interarm regions) is important when considering the triggers and thresholds of star formation.

The classic scenario of rapid phase transition is supported by observations of MCs in the solar neighborhood, the Large Magellanic Cloud (LMC), and the nearby spiral galaxy M33. Local Galactic MCs linked with OB stars appear photodissociated (e.g., Tachihara et al. 2001). Young star clusters often show no parent MCs nearby as the cluster ages approach $\sim 10-40 \mathrm{Myr}$, and the MCs may be destroyed by stellar feedback (Hartmann et al. 2001; Kawamura et al. 2009; Miura et al. 2012). Molecular gas and clouds exist primarily within HI

\footnotetext{
4 Following Solomon \& Edmunds (1980) and Heyer \& Dame (2015), we use the term "MCs" for all clouds (small and large) whereas the term "giant MCs" is used for clouds with $>10^{5} M_{\odot}$.
}

spiral arms with little or no $\mathrm{H}_{2}$ gas in the interarm regions (Engargiola et al. 2003; Heyer et al. 2004; Fukui et al. 2009). Thus, the MC lifetimes should be as short as an arm crossing time $30 \mathrm{Myr}$. The molecular gas should therefore be dissociated after spiral arm passages.

Early interferometric observations of the grand-design spiral galaxy M51 showed an apparent absence of molecular gas in its interarm regions (Vogel et al. 1988; Rand \& Kulkarni 1990) because it was largely resolved out. High-fidelity imaging of molecular gas is necessary to fully reveal the ISM evolution in both spiral arms and interarm regions (Koda et al. 2011; Pety et al. 2013). The dynamically driven scenario is built on two observational facts from high-fidelity imaging. In interarm regions, molecular emission is abundant compared with atomic emission, and their ratio shows little gas-phase change between the arm and interarm regions. The MC mass spectrum, however, changes between the two regions, and the most massive MCs are present only in the spiral arms. The coagulation and fragmentation of MCs without the dissociation of molecules could naturally explain these two facts (Koda et al. 2009). More recent CO(1-0) observations of M51's central region (Schinnerer et al. 2013) also confirmed the change of the MC mass spectrum between arm and interarm regions (Colombo et al. 2014). They re-confirmed that stellar feedback is not the dominant MC destruction mechanism, since star forming regions are too localized. Such dynamically driven ISM evolution is supported by numerical simulations (e.g., Kwan \& Valdes 1987; Wada \& Koda 2004; Dobbs et al. 2006; Wada 2008; Tasker \& Tan 2009).

In this paper, we quantify the fraction of molecular gas over total neutral gas $\left(\mathrm{HI}+\mathrm{H}_{2}\right)$ in the MW and study its azimuthal and radial variations. The molecular fraction shows a clear radial decrease from the molecule-dominated center to the 
atom-dominated outskirts. The azimuthal variations depend on the globally dominant phase: in the molecule-dominated inner region $(R \lesssim 6 \mathrm{kpc})$, the phase changes only marginally, by only $\sim 20 \%$, during spiral arm passages. However, in the atomdominated outskirts $(R \gtrsim 6 \mathrm{kpc})$, the phase change is more pronounced.

\subsection{Brief History}

Historically, ISM evolution was often discussed in the context of MC lifetimes and distribution; it was debated intensely in the 1980s with no consensus emerging (e.g., Scoville \& Hersh 1979; Blitz \& Shu 1980). In fact, two major Galactic plane $\mathrm{CO}(1-0)$ surveys arrived at contradictory conclusions. The Columbia/Harvard survey found very little $\mathrm{CO}$ emission in the interarm regions of the longitude-velocity $(l-v)$ diagram (LVD), which was interpreted as meaning that MCs survive only for the duration of spiral arm passage (of the order of 20-30 Myr; Blitz \& Shu 1980; Cohen et al. 1980). On the other hand, the Massachusett-Stony Brook survey found an abundant population of GMCs even in the interarm regions, and so concluded that GMCs live longer than the arm-to-arm transit timescale ( $>100 \mathrm{Myr}$; Sanders et al. 1985; Scoville \& Wilson 2004).

The discussion continues with more recent ${ }^{13} \mathrm{CO}(J=1-0)$ data (Jackson et al. 2006). Roman-Duval et al. (2009, 2010) found MCs only on spiral arms in their face-on map of the MW, implying a short cloud lifetime $(<10 \mathrm{Myr}$; their arm crossing time). Their analysis, however, suffered from the ambiguity problem for kinematic distance (see Section 3.2). Indeed, Roman-Duval et al. (2010) found MC concentrations between the Sagittarius-Carina and Perseus arms, but considered them to be clouds in the Perseus arm due to possible errors in the distance estimate. Using the same data, Koda et al. (2006) found MCs in the interarm regions using an analysis of the LVD, which does not involve the distance estimate. The spiral arm locations in the LVD, however, are still debatable.

Further progress requires an analysis that does not rely on spiral arm locations or on a distance estimate. In this paper, we develop such an analysis.

\subsection{Structures of the $M W$}

The MW is a barred spiral galaxy with a stellar exponential disk scale length of $3.9 \pm 0.6 \mathrm{kpc}$ (Benjamin et al. 2005). The presence of the central bar was confirmed by stellar distributions. It has a half-length of $4.4 \pm 0.5 \mathrm{kpc}$ with a tilt of $44^{\circ} \pm 10^{\circ}$ to the Sun-Galactic center line (Benjamin et al. 2005). This corresponds to Galactic longitudes from $l=345^{\circ}$ (or $-15^{\circ}$ ) to $30^{\circ}$. Figure 1 shows a conceptual image of the MW in face-on projection (Churchwell et al. 2009) and its spiral arms.

Uncertainties still remain as to the precise number and geometry of spiral arms, with results differing depending on the adopted tracer (ISM versus stars). Four spiral arms are often inferred when gas, dust, or star forming regions are used as tracers of spiral structure (e.g., Georgelin \& Georgelin 1976; Paladini et al. 2004; Steiman-Cameron et al. 2010, Hou \& Han 2014; Vallée 2014). On the other hand, only two arms are indicated by stellar distributions (Drimmel \& Spergel 2001; Benjamin 2008; Churchwell et al. 2009). Robitaille et al. (2012) argued for two major and two minor arms from a jointanalysis of stellar and gas/dust distributions. An interpretation of two spiral arms with other two ISM filaments seems most reasonable, as barred spiral galaxies typically have only two prominent arms. The ISM in galaxies often shows coherent filamentary structures, even in interarm regions (seen as dust lanes in optical images; e.g., Hubble Heritage images-http:// heritage.stsci.edu), which explains the minor, apparent gas/ dust spiral arms. In addition, gas filaments in the interarm regions of stellar spiral arms develop naturally in numerical simulations (e.g., Kim \& Ostriker 2002; Chakrabarti et al. 2003; Martos et al. 2004; Wada \& Koda 2004; Dobbs \& Bonnell 2006; Pettitt et al. 2015).

Here, we adopt the determination of the spiral arms provided by Benjamin (2008): the Perseus and Scutum-Crux (or ScutumCentaurus) arms are the two major spiral arms associated with the stellar spiral potential. The Sagittarius-Carina and NormaCygnus features are the two minor spiral arms without stellar counterparts. The four arms are drawn in Figure 1. We adopt the parameters from Benjamin et al. (2005) and Churchwell et al. (2009) because of the overall consistency of the bar and spiral arm structures. We note that there are more recent studies of the MW's structures (e.g., Francis \& Anderson 2012; Robin et al. 2012; Wegg et al. 2015). For example, in Figure 1, the inner part of the Perseus arm passes the far end of the bar and overlaps with the near $3 \mathrm{kpc}$ arm (see Churchwell et al. 2009). This section is drawn based on the assumption of symmetry with the Scutum-Crux arm, but is debatable (e.g., Vallée 2016). In any case, our discussion does not depend on the precise definition of the structures.

\section{DATA}

In this study, archival $\mathrm{HI} 21 \mathrm{~cm}$ and $\mathrm{CO}(J=1-0)$ emission data are used for the analysis of the neutral gas phases. We focus on the inner part of the MW within the Solar radius: the range of Galactic longitude $l$ from $0^{\circ}$ to $90^{\circ}$ (the northern part of the inner $\mathrm{MW}$ disk) and from $270^{\circ}$ to $360^{\circ}$ (or equivalently $-90^{\circ}$ to $0^{\circ}$; the southern part). For most discussions in this paper, we integrate emission within the Galactic latitude $b$ from $-30^{\circ}$ to $+30^{\circ}$, which covers the full thickness of the gas disk (Section 4.1). To determine the properties at the Galactic midplane, emission is integrated over $-1.5<b<+1.5$.

The HI $21 \mathrm{~cm}$ line emission data (three-dimensional data cube) is taken from the Leiden-Argentine-Bonn (LAB) survey (Kalberla et al. 2005). The LAB survey is the combination of the Leiden/Dwingeloo survey (Hartmann \& Burton 1997) and the Instituto Argentino de Radioastronomia Survey (Arnal et al. 2000; Bajaja et al. 2005). The data are corrected for stray radiation picked up by the antenna sidelobes, and therefore provide excellent calibration of the $21 \mathrm{~cm}$ line emission. These archival data cover the entire sky from -450 to $+450 \mathrm{~km} \mathrm{~s}^{-1}$ at 0.6 and $1.3 \mathrm{~km} \mathrm{~s}^{-1}$ resolutions with a spatial pixel size of 0.5 . From the data, the root-mean-square (rms) noise on the main beam temperature scale is estimated to be $0.07-0.09 \mathrm{~K}$.

The $\operatorname{CO}(J=1-0)$ data is from the Columbia/CfA survey (Dame et al. 2001). This compilation of 37 individual surveys covers all regions with relatively high dust opacities (Planck Collaboration et al. 2011), and hence includes virtually all of the $\mathrm{CO}$ emission in the MW. The angular resolution and pixel size are $8^{\prime}\left(\sim 0^{\circ} .13\right)$ and $0^{\circ} .125$, respectively. The spatial sampling is not uniform and ranges from a full-beam $\left(0^{\circ} .125\right)$ in the Galactic plane to a super-beam $(0.5)$ at higher latitudes. This does not significantly affect the analyses since the CO emission resides predominantly in the thin midplane 


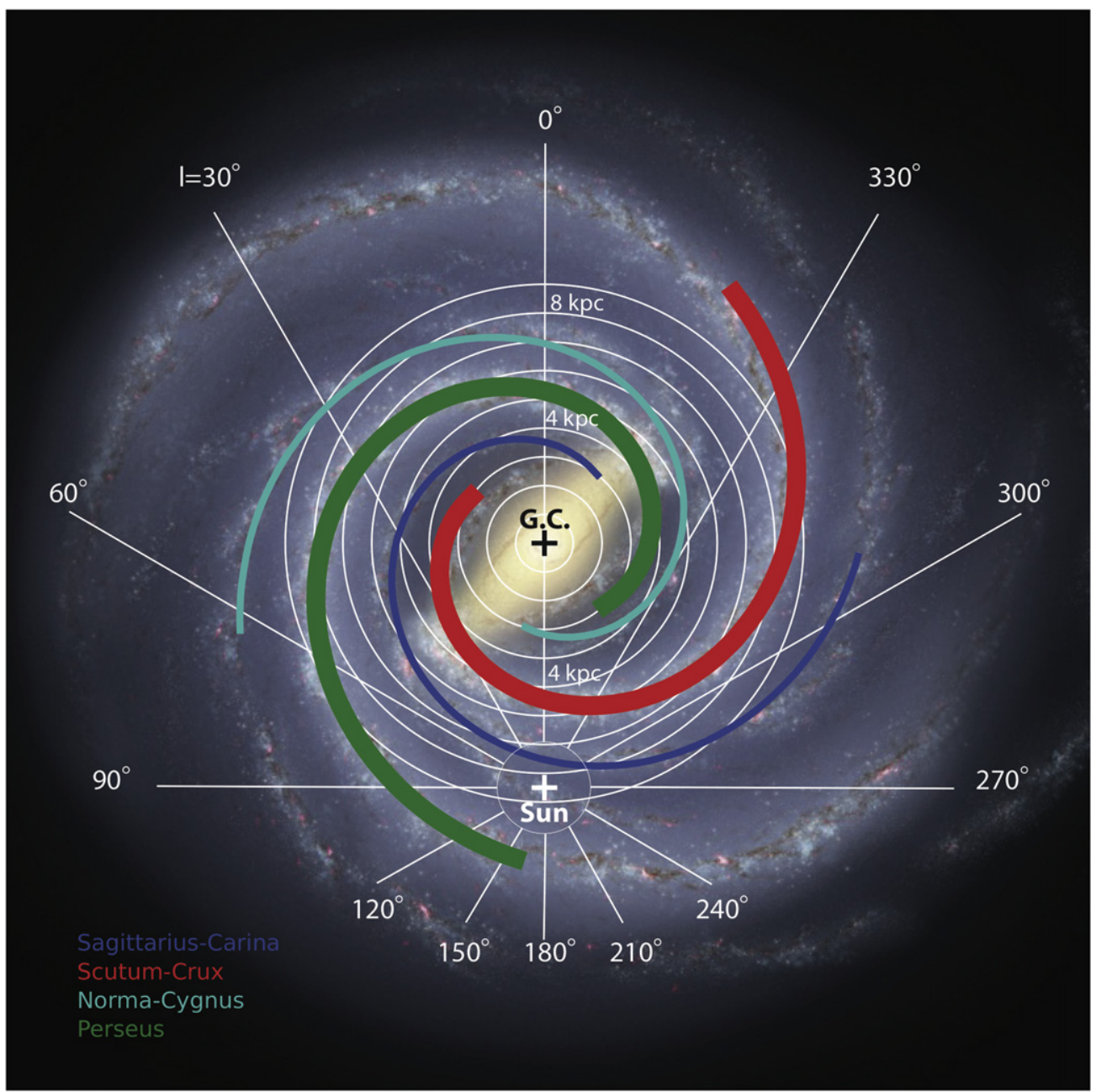

Figure 1. Schematic illustration of the face-on view of the Milky Way. The illustration in the background is from Churchwell et al. (2009). The logarithmic spiral arms that we adopted in this paper for qualitative discussions are overplotted. The blue, red, cyan, and green correspond to the Sagittarius-Carina, Scutum-Crux (aka Scutum-Centaurus), Norma-Cygnus, and Perseus arms, respectively. Four spiral arms are indicated when tracers of the interstellar medium (gas or dust) or star formation are used, while only two arms, Scutum-Crux and Perseus, are traced by the enhancement of stars (see the text). Concentric circles around the Galactic Center (G.C.) are drawn at a $1 \mathrm{kpc}$ interval.

$\left(|b| \lesssim 5^{\circ}\right.$; see Section 4.1$)$ where the sampling is typically 0.125 . The velocity resolution is $1.3 \mathrm{~km} \mathrm{~s}^{-1}$. The rms noise is $0.12-0.31 \mathrm{~K}$ for the inner MW.

To match resolutions, the $\mathrm{CO}$ data are smoothed to a 0.6 resolution and regridded to a 0.5 pixel size. The total integrated flux is conserved in these operations. Both $\mathrm{HI}$ and $\mathrm{CO}$ cubes are then binned at a $6.2 \mathrm{~km} \mathrm{~s}^{-1}$ velocity resolution. The width of the velocity bin is chosen so that local fluctuations, e.g., due to MCs with typical velocity dispersions of $\sim 4 \mathrm{~km} \mathrm{~s}^{-1}$ (for giant MCs; Scoville et al. 1987), are smoothed out, but the spiral arms and interarms are still resolved (see Section 3.3 and Appendix). The spatial resolution of 0.6 corresponds to a linear scale of $104(d / 10 \mathrm{kpc}) \mathrm{pc}$ for the heliocentric distance $d$ in kiloparsecs. Note that we integrate the data over a larger range in Galactic latitude (b). Figures 2(a) and (b) show the LVDs of the $\mathrm{HI}$ and $\mathrm{CO}$ emission. The smoothed data cubes are integrated over $-30^{\circ}<b<+30^{\circ}$ for these figures. Figure 3 is the same as Figure 2, but shows a zoom-in on the inner MW.

\section{METHOD}

Our goal is to analyze variations of the molecular gas fraction $f_{\text {mol }}$ (defined in Section 3.1) in the radial and azimuthal directions. In this section, we first calculate $f_{\text {mol }}$ in an LVD (Section 3.1; Figure 3(c)), mapping each $(l, v)$ pixel into a Galactcentric radius $R$ based on the assumption of Galactic circular rotation (Section 3.2), and then producing an $f_{\mathrm{mol}}-R$ plot (Figure 4).

This direct conversion of $f_{\mathrm{mol}}$ in an LVD to that in $R$ has clear advantages. First, it does not require heliocentric distances, since $f_{\text {mol }}$ is a distance-independent parameter. A more conventional analysis of MW structures resolves the near-far distance ambiguity in kinematic distance measurements. While this offers a measure of the $\mathrm{CO}$ or $\mathrm{HI}$ luminosities, and therefore the mass, it can also generate systematic uncertainties. Second, the LVD samples both spiral arms and interarm regions (Section 3.3), even though their exact locations are uncertain. The $f_{\mathrm{mol}}-R$ plot (Figure 4) should therefore include data from both arm and interarm regions. Therefore, variations of $f_{\text {mol }}$ at a fixed radius in this plot correspond to azimuthal variations, i.e., arm/interarm variations, at that radius. In this manner, the radial and azimuthal variations of $f_{\mathrm{mol}}$ are quantified without identifying the exact locations of the spiral arms and interarm regions. 

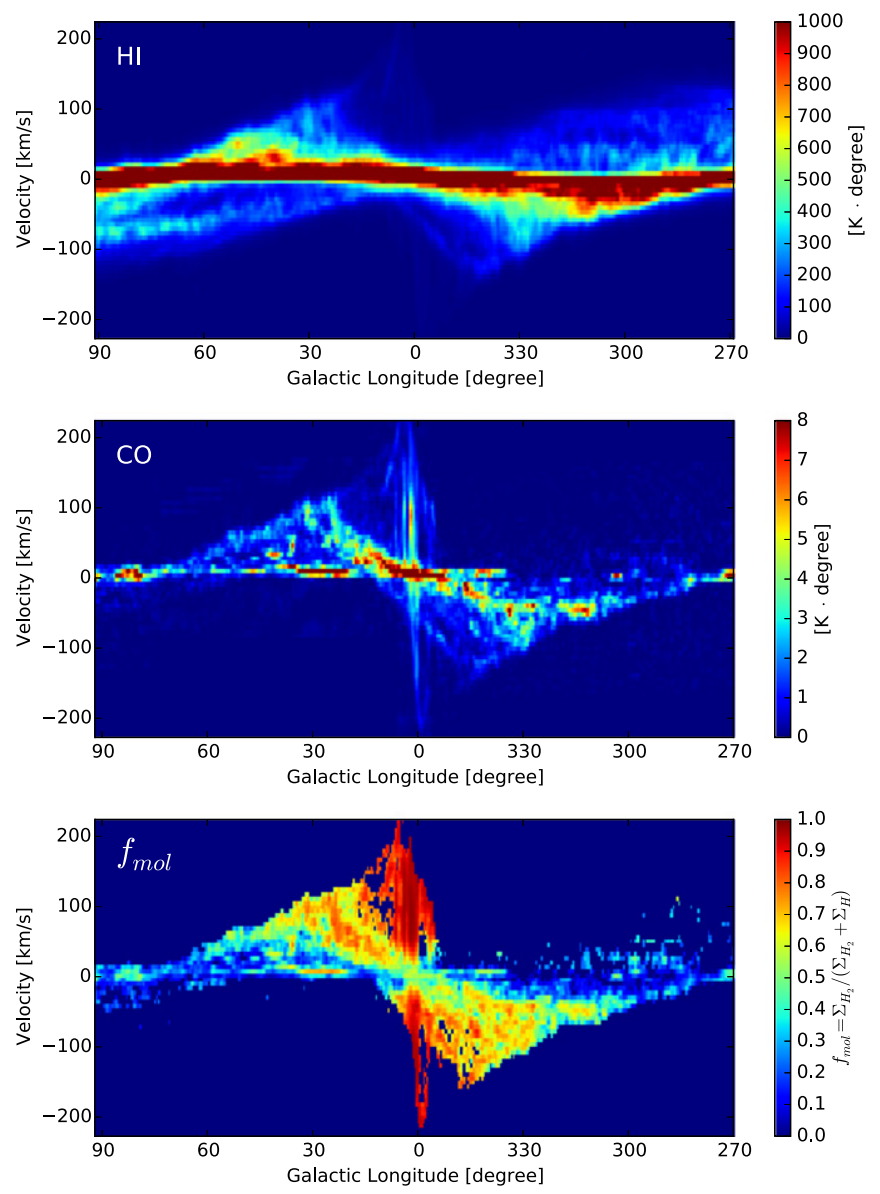

Figure 2. Galactic longitude-velocity ( $l-v)$ diagrams (LVDs) of the (a) HI $21 \mathrm{~cm}$ data, (b) CO $J=1-0$ data, and (c) molecular fraction $f_{\text {mol }}$. The $y$ axis is the Local Standard of Rest (LSR) velocity. The data are smoothed to 0.5 and $6.2 \mathrm{~km} \mathrm{~s}^{-1}$ resolutions and integrated over Galactic latitude $-30^{\circ}<b<+30^{\circ}$. We adopt a CO-to- $\mathrm{H}_{2}$ conversion factor of $X_{\mathrm{CO}}=$ $2 \times 10^{20} \mathrm{~cm}^{-2}\left(\mathrm{~K} \mathrm{~km} \mathrm{~s}^{-1}\right)^{-1}$.

Potential errors in our analysis are sumarized in Section 4.5, and here we note one caveat. Each velocity at a given $l$ corresponds to two locations along the line of sight (i.e., near and far sides; see Section 3.2). They are averaged in the $f_{\text {mol }}$ calculation. This is not a problem where both the near and far sides correspond to either arm or interarm regions. An LVD shows two types of regions (see Section 3.3): one in which both the near and far sides are interarm regions, and the other where a spiral arm and an interarm region overlap. For the latter case, spiral arm emission is most likely dominant, and their average should represent the $f_{\text {mol }}$ of the spiral arm.

\subsection{The Molecular Fraction}

The azimuthally averaged radial trend of gas phase in galaxies has been studied with two different expressions for the fraction of molecular gas (Elmegreen 1993; Sofue et al. 1995; Wong \& Blitz 2002; Blitz \& Rosolowsky 2006). We use the definition adopted by Sofue et al. (1995). The molecular fraction $f_{\text {mol }}$, i.e., the mass fraction of $\mathrm{H}_{2}$ gas over the total $\mathrm{HI}$ $+\mathrm{H}_{2}$ gas, is expressed as

$$
f_{\mathrm{mol}} \equiv \frac{\Sigma_{\mathrm{H}_{2}}}{\Sigma_{\mathrm{H}_{2}}+\Sigma_{\mathrm{HI}}}
$$

where $\Sigma_{\mathrm{H}_{2}}$ and $\Sigma_{\mathrm{HI}}$ are the surface densities of the $\mathrm{H}_{2}$ and $\mathrm{HI}$ gas, respectively. The $\Sigma_{\mathrm{H}_{2}}$ and $\Sigma_{\mathrm{HI}}\left(M_{\odot} \mathrm{pc}^{-2}\right)$ are calculated from the $\mathrm{HI}$ and $\mathrm{CO}$ integrated intensities, $I_{\mathrm{HI}}$ and $I_{\mathrm{CO}}$ $\left(\mathrm{K} \mathrm{km} \mathrm{s}^{-1}\right)$, at each $(l, v)$ pixel, respectively. Based on the assumption of optically thin HI $21 \mathrm{~cm}$ emission,

$$
\Sigma_{\mathrm{HI}}=1.45 \times 10^{-2} I_{\mathrm{HI}}\left(\frac{d v}{d d}\right)
$$

where the expression in parentheses is a derivative of $v$ with respect to $d$. Using the CO-to- $\mathrm{H}_{2}$ conversion factor $X_{\mathrm{CO}}$, we have

$$
\Sigma_{\mathrm{H}_{2}}=3.2 I_{\mathrm{CO}}\left(\frac{X_{\mathrm{CO}}}{2 \times 10^{20} \mathrm{H}_{2} \mathrm{~cm}^{-2}\left(\mathrm{~K} \mathrm{~km} \mathrm{~s}^{-1}\right)^{-1}}\right)\left(\frac{d v}{d d}\right) .
$$

We use $X_{\mathrm{CO}}=2 \times 10^{20} \mathrm{H}_{2} \mathrm{~cm}^{-2}\left(\mathrm{~K} \mathrm{~km} \mathrm{~s}^{-1}\right)^{-1}$ as recommended by Bolatto et al. (2013); $f_{\text {mol }}$ increases by $\sim 10 \%$ at some radii if an $X_{\mathrm{CO}}$ of $3 \times 10^{20} \mathrm{H}_{2} \mathrm{~cm}^{-2}\left(\mathrm{~K} \mathrm{~km} \mathrm{~s}^{-1}\right)^{-1}$ is adopted instead (see Section 3.1.1). The " $d v / d d$ " term is the lineof-sight velocity gradient and converts $I_{\mathrm{HI}}$ and $I_{\mathrm{CO}}$ in a velocity bin to the values in a face-on projection of the MW disk (e.g., Nakanishi \& Sofue 2003, 2006). Our analysis does not suffer from systematic uncertainty due to this term because it cancels out in Equation (1). We do not include the $\mathrm{He}$ mass in Equations (2) and (3) because it also cancels out in Equation (1).

Figures 2(c) and 3(c) show $f_{\mathrm{mol}}$ in LVDs. The sensitivities in $\mathrm{HI}$ and $\mathrm{CO}$ change within the diagrams. Our analysis is limited by the sensitivity of the $\mathrm{CO}$ observations. We roughly derived a conservative rms noise estimate from emission-free pixels and cut the pixels below $\sim 3 \sigma$ significance, i.e., $0.17 \mathrm{~K}$ degree for CO. The sensitivity should be higher in the inner part that we analyze in this study. The HI emission is detected much more significantly at all locations, and for Figure 2 we imposed the cut-off at a threshold surface density $\sim 7$ times lower than that for $\mathrm{CO}$. The pixels with low signal to noise are blanked in these figures and are not included in the subsequent analysis. This excludes virtually no point between the Galactocentric radii $3.5 \lesssim R \lesssim 8.5 \mathrm{kpc}$, but does exclude some at $R \lesssim 3.5 \mathrm{kpc}$ (see Section 3.2). The removed points correspond to the radii inside those of the Galactic bar, but azimuthally not in the bar. Gas is often at a deficit there in other barred galaxies (e.g., Sheth et al. 2002).

\subsubsection{Notes on Dark Gas and $X_{\mathrm{CO}}$}

The presence of dark gas, invisible in $\mathrm{CO}$ or $\mathrm{HI}$ emission, is inferred from $\gamma$-ray, submillimeter surveys of dust emission and far-IR spectroscopy of [C II] emission (Grenier et al. 2005; Planck Collaboration et al. 2011; Pineda et al. 2013). Its mass fraction is estimated to be about $22 \%$ in the solar neighborhood. This gas could be CO-deficient $\mathrm{H}_{2}$ gas (van Dishoeck \& Black 1988; Wolfire et al. 2010) or optically thick HI gas (Fukui et al. 2014, 2015), and in reality may be a mixture of both. Our analysis does not include the dark gas, but the dark $\mathrm{H}_{2}$ and $\mathrm{HI}$ gas should compensate for each other in the $f_{\mathrm{mol}}$ calculation if their fractions are similar.

The actual $X_{\mathrm{CO}}$ in the MW disk could be larger than that recommended by Bolatto et al. (2013). Among all of the measurements, only two, those based on virialized MCs and $\gamma$ ray emission, attempt to anchor their calibrations using actual $\mathrm{HI}+\mathrm{H}_{2}$ mass measurements. The others rely on scalings with 

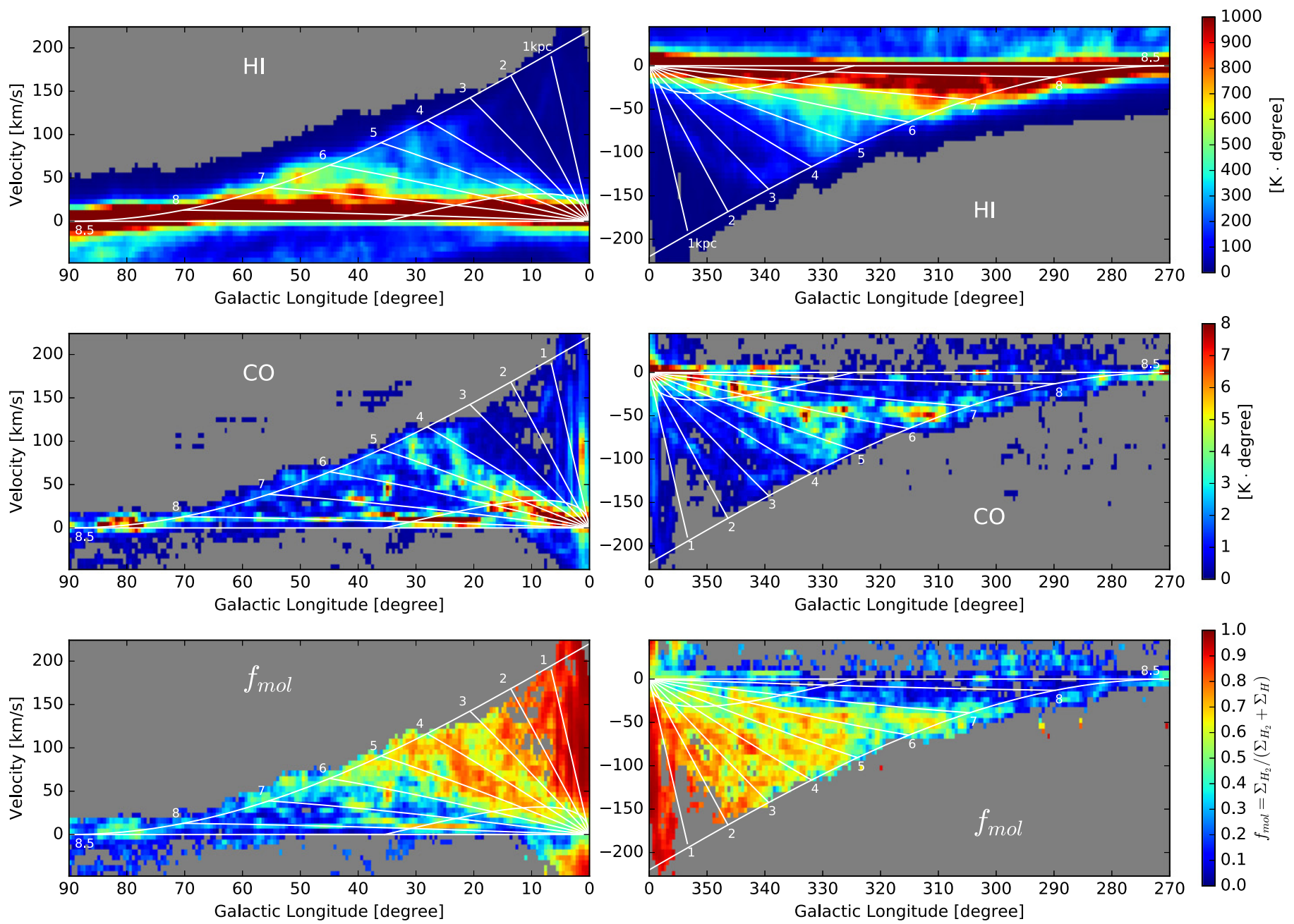

Figure 3. Same as Figure 2, but zoomed in on the inner MW. The northern part (left panels) and southern part of the Galactic plane (right panels) are separated Overplotted are maximum velocity (tangent velocity) curves, as well as Galactocentric distance lines from $d=1$ to $8 \mathrm{kpc}$ with a $1 \mathrm{kpc}$ interval, except for the outermost one at $8.5 \mathrm{kpc}$. $f_{\text {mol }}$ clearly decreases from the Galactic center to the outer part. The other curves, the arcs from the origins to around $l \sim 35^{\circ}$, are the boundaries where the deviations of $\pm 15 \mathrm{~km} \mathrm{~s}^{-1}$ from a circular rotation result in errors of $1 \mathrm{kpc}$ in $R$; we remove the data below these $|v|$ to avoid large errors.

abundances or gas-to-dust ratios without measuring the mass. Those scaling constants are at least as uncertain as $X_{\mathrm{CO}}$. The average value among virialized $\mathrm{MCs}$ is $X_{\mathrm{CO}}=3.6 \times 10^{20}$ (Scoville et al. 1987) or $3.5 \times 10^{20}$ (Solomon et al. 1987, using $R_{0}=8.5 \mathrm{kpc}$ ), instead of the value that Bolatto et al. (2013) derived for a typical GMC from the same data (we omit the unit, $\left.\mathrm{H}_{2} \mathrm{~cm}^{-2}\left(\mathrm{~K} \mathrm{~km} \mathrm{~s}^{-1}\right)^{-1}\right)$. The background cosmic-ray distribution for $\gamma$-ray production is still uncertain, and $X_{\mathrm{CO}}$ from $\gamma$-rays suffers from this uncertainty. There is also the possibility that $X_{\mathrm{CO}}$ is smaller in the central molecular zone of the MW. The following equation converts $f_{\mathrm{mol}}$ with $X_{\mathrm{CO}}=2 \times 10^{20}$ to the value $f_{\mathrm{mol}}^{\prime}$ with some other $X_{\mathrm{CO}}^{\prime}$ :

$$
f_{\mathrm{mol}}^{\prime}=\frac{\left(X_{\mathrm{CO}}^{\prime} / X_{\mathrm{CO}}\right) f_{\mathrm{mol}}}{\left(X_{\mathrm{CO}}^{\prime} / X_{\mathrm{CO}}\right) f_{\mathrm{mol}}+\left(1-f_{\mathrm{mol}}\right)} .
$$

Table 1 presents $f_{\text {mol }}$ using the consensus $X_{\mathrm{CO}}$ value and a slightly larger one (see Section 4.2). The differences do not affect the main conclusions of this paper.

\subsection{The Constant Circular Rotation Model}

Under the assumption of constant circular rotation of the $\mathrm{MW}$, a simple geometric consideration provides the equation for conversion from $(l, v)$ to $R$ (e.g., Oort et al. 1958; Kellermann \& Verschuur 1988; Nakanishi \& Sofue 2003):

$$
R=R_{0} \frac{V_{0} \sin l}{v+V_{0} \sin l},
$$

where we set the constant rotation velocity to $V_{0}=220 \mathrm{~km} \mathrm{~s}^{-1}$ and the Sun's location at $R_{0}=8.5 \mathrm{kpc}$. This equation is applicable in a particular velocity range at each $l$ (explained later): in summary, $0 \leqslant v \leqslant V_{0}(1-\sin l)$ for $0 \leqslant l \leqslant 90^{\circ}$ and $-V_{0}(1+\sin l) \leqslant v \leqslant 0$ for $270 \leqslant l \leqslant 360^{\circ}$. Figure 3 shows LVDs with lines of constant $R$ at $1 \mathrm{kpc}$ intervals and at $R=8.5 \mathrm{kpc}$. Figure 4(a) shows the $f_{\mathrm{mol}}-R$ plot from Figure 3(c), integrated over the whole gas disk thickness $\left(|b|<30^{\circ}\right.$; Section 4.1), and Figure 4(b) is for the Galactic midplane $|b|<1.5$.

We do not use the heliocentric distance $d$, except for the calculation of vertical profiles of the molecular and atomic gas at tangent points (defined below). $d$ and $R$ are related by

$$
d=R_{0} \cos l \pm \sqrt{R^{2}-R_{0}^{2} \sin ^{2} l}
$$



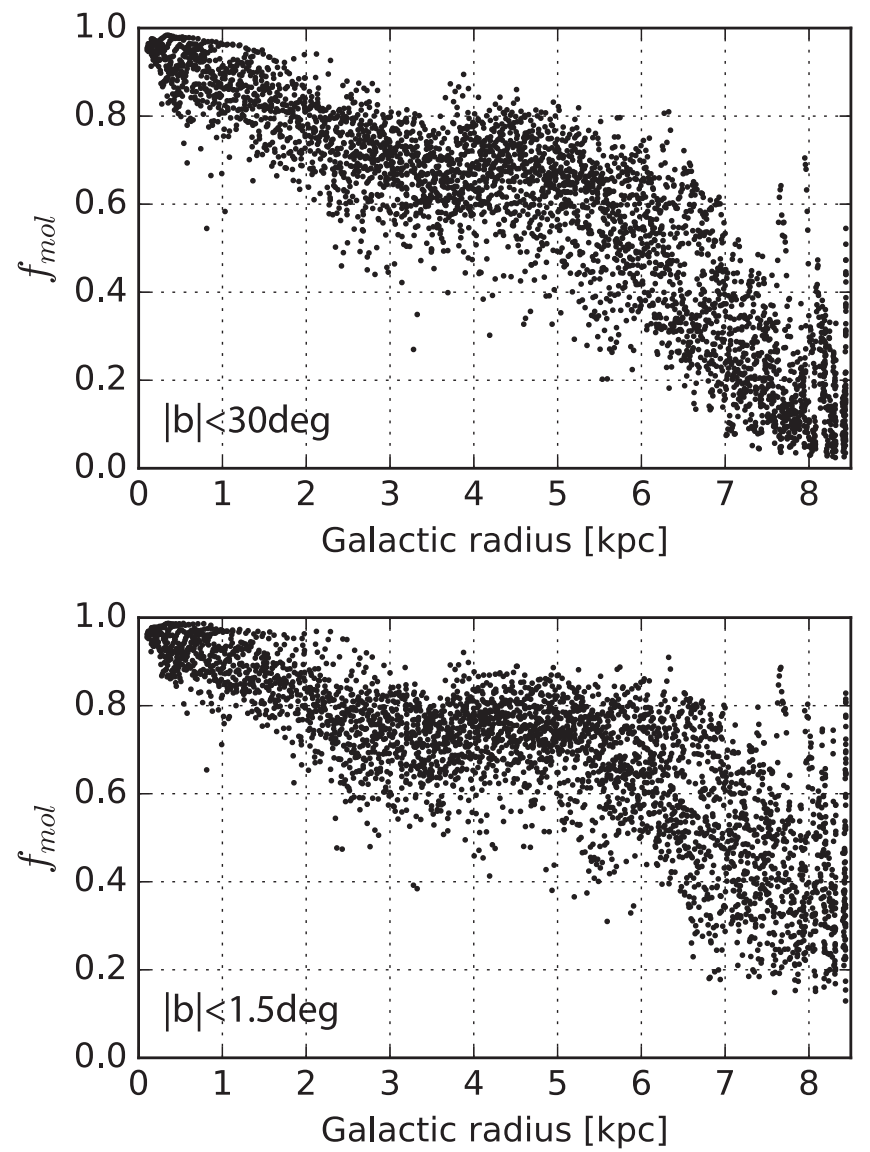

Figure 4. Molecular fraction $f_{\text {mol }}$ as a function of $R$. The atomic and molecular gas is integrated (a) over the whole disk thickness $|b|<30^{\circ}$ and (b) around the midplane $|b|<1^{\circ} .5$. The coordinates $(l, v)$ of each pixel in the LVD (Figure 3) are converted to $R$ using Equation (5). The radially decreasing trend is clear in both panels. The scatter at each radius represents variations of $f_{\mathrm{mol}}$ along the circular orbit at that Galactocentric radius, therefore showing the amount of azimuthal variations, and hence arm/interarm variations.

for the inner MW (i.e., $R<R_{0}$ ). Two solutions, " \pm " for the near and far distances, are possible for a given $R$, and hence for a given $(l, v)$; this is the near-far distance ambiguity.

The line of sight at $l$ is tangential to the circular orbit of radius $R=R_{0}|\sin l|$. Their intersection is called a tangent point. The line-of-sight velocity $v$ in the direction of $l$ takes its maximum absolute value at the tangent point,

$$
v= \begin{cases}V_{0}(1-\sin l) & \left(0 \leqslant l \leqslant 90^{\circ}\right) \\ -V_{0}(1+\sin l) & \left(270 \leqslant l \leqslant 360^{\circ}\right),\end{cases}
$$

which set the velocity range for Equation (5). These maximum line-of-sight velocities (i.e., tangent velocities) are drawn in Figure 3. There is no distance ambiguity problem at tangent points ( $d=R_{0} \cos l$ from Equation (6)). Figure 5(b) shows the vertical profiles of the $\mathrm{HI}$ and $\mathrm{H}_{2}$ gas masses at the tangent points, which is discussed in Section 4.1.

\subsubsection{Removal of Regions of Potentially Large Errors}

The constant $R$ lines are crowded around small $|l|$ and $|v|$ in Figure 3. We remove these regions since a small deviation from circular rotation would result in a large error in $R$. By taking the derivative of Equation (5) we derive the relation between a slight shift $\Delta v$ and the resultant error $\Delta R$. We then obtain the equation for removal:

$$
\left|\frac{\Delta R}{\Delta v}\right|=\left|\frac{R_{0} V_{0} \sin l}{\left(v+V_{0} \sin l\right)^{2}}\right|>\gamma .
$$

Arbitrarily, we set $\gamma=1 / 15\left(\mathrm{kpc}\left(\mathrm{km} \mathrm{s}^{-1}\right)^{-1}\right)$, which means that an $(l, v)$ pixel is removed if a $15 \mathrm{~km} \mathrm{~s}^{-1}$ deviation in $v$ from circular rotation causes an error larger than $1 \mathrm{kpc}$ in $R$. These threshold lines are drawn in Figure 3 as arcs that start from the origin and run to about $l \sim \pm 35^{\circ}$; the pixels with smaller $|l|$ and $|v|$ are removed.

\subsection{Spiral Arms and Non-circular Motions}

Gas motions are approximated using circular rotation in our analysis, and we do not include non-circular motions associated with the MW spiral arms. The spiral arms and associated noncircular motions affect the LVD in a systematic way. Their impact is small, as demonstrated in Appendix A. In this model, two points are important to keep in mind.

First, the locations of the spiral arms systematically shift in the velocity direction on an LVD due to non-circular motions (the "loops" of spiral arms in LVD are squashed in the velocity direction). This does not change the areas that the spiral arms occupy in an LVD, but causes errors in $R$ if it is determined while assuming circular rotation. This results in an artificial steepening of the radial gradient of $f_{\text {mol }}$ near the tangent radii of the spiral arms. (See Figures 12(c) and (d) in the Appendix-if the black solid lines represent the intrinsic radial profile of $f_{\mathrm{mol}}$, then the error vectors show how the points on the profile apparently shift due to the non-circular motions. By connecting the vector heads, we see an apparently steepened radial profile. The vector directions change from right to left near the tangents of the spiral arms.)

Second, even with increased velocity widths due to enhanced velocity dispersions and spiral arm streaming, interarm regions are still sampled in the LVD (Figure 6). Thus, the $f_{\mathrm{mol}}-R$ plot from the LVD includes data from both spiral arms and interarm regions.

\section{RESULTS}

\subsection{Vertical Profiles}

Figure 5 shows the vertical profiles of the atomic (HI) and molecular $\left(\mathrm{H}_{2}\right)$ gas masses at tangent points at $15^{\circ}$ intervals in $l$. The amplitude scales for the $\mathrm{HI}$ and $\mathrm{H}_{2}$ profiles are the same in mass density within each panel; however, different scalings are used in the different panels. Regions of $\pm 5^{\circ}$ in $l$ and $\pm 20 \mathrm{~km} \mathrm{~s}^{-1}$ in $v$ are integrated at the tangent points (i.e., at the terminal velocities). Note that the Galactocentric distance $R$ increases from low to high $l$. Panel (a) shows the distributions as a function of Galactic latitude $b$. Both $\mathrm{HI}$ and $\mathrm{CO}$ are confined within $-30^{\circ}<b<30^{\circ}$, over which we integrate the $\mathrm{HI}$ and CO emission for the LVDs (except for Figure 4(b) where only the midplane $-1.5<b<1.5$ is integrated).

On a parsec scale, the molecular gas is confined in the thin midplane at all $l$ (and $R$ ), while the atomic gas is distributed over a thicker disk. Figure 5(b) shows the vertical distributions with the Galactic altitude $Z$ from the midplane in parsecs. The FWHM thicknesses of the atomic and molecular disks within the Solar radius $(R=8.5 \mathrm{kpc})$ were measured using functional fits (e.g., Gaussian or $\operatorname{sech}^{2}$ ) and are $\sim 100-200 \mathrm{pc}$ and $\sim 50-100$ pc, respectively (Sanders et al. 1984; Dickey \& 
Table 1

Molecular Fraction $f_{\text {mol }}$

\begin{tabular}{|c|c|c|c|c|c|c|}
\hline \multirow{2}{*}{$\begin{array}{l}R \\
\text { (kpc) }\end{array}$} & \multicolumn{3}{|c|}{ Midplane } & \multicolumn{3}{|c|}{ Whole Disk Thickness } \\
\hline & North & South & Average & North & South & Average \\
\hline & \multicolumn{6}{|c|}{$X_{\mathrm{CO}}=2 \times 10^{20} \mathrm{~cm}^{-2}\left(\mathrm{~K} \mathrm{~km} \mathrm{~s}^{-1}\right)^{-1}$} \\
\hline 0.0 & $0.96 \pm 0.03$ & $0.92 \pm 0.03$ & $0.94 \pm 0.04$ & $0.95 \pm 0.04$ & $0.91 \pm 0.04$ & $0.93 \pm 0.04$ \\
\hline 1.0 & $0.91 \pm 0.05$ & $0.88 \pm 0.06$ & $0.90 \pm 0.06$ & $0.89 \pm 0.06$ & $0.84 \pm 0.07$ & $0.87 \pm 0.07$ \\
\hline 2.0 & $0.84 \pm 0.07$ & $0.77 \pm 0.08$ & $0.81 \pm 0.08$ & $0.80 \pm 0.07$ & $0.74 \pm 0.08$ & $0.77 \pm 0.08$ \\
\hline 3.0 & $0.74 \pm 0.08$ & $0.71 \pm 0.09$ & $0.72 \pm 0.08$ & $0.69 \pm 0.08$ & $0.67 \pm 0.09$ & $0.68 \pm 0.09$ \\
\hline 4.0 & $0.74 \pm 0.08$ & $0.71 \pm 0.08$ & $0.73 \pm 0.08$ & $0.68 \pm 0.08$ & $0.65 \pm 0.09$ & $0.67 \pm 0.09$ \\
\hline 5.0 & $0.74 \pm 0.10$ & $0.70 \pm 0.10$ & $0.72 \pm 0.10$ & $0.66 \pm 0.11$ & $0.61 \pm 0.10$ & $0.63 \pm 0.11$ \\
\hline 6.0 & $0.61 \pm 0.10$ & $0.70 \pm 0.11$ & $0.65 \pm 0.11$ & $0.46 \pm 0.12$ & $0.57 \pm 0.12$ & $0.51 \pm 0.13$ \\
\hline 7.0 & $0.49 \pm 0.14$ & $0.54 \pm 0.17$ & $0.52 \pm 0.16$ & $0.29 \pm 0.12$ & $0.36 \pm 0.16$ & $0.32 \pm 0.15$ \\
\hline \multirow[t]{2}{*}{8.0} & $0.46 \pm 0.17$ & $0.37 \pm 0.13$ & $0.43 \pm 0.16$ & $0.23 \pm 0.14$ & $0.13 \pm 0.07$ & $0.19 \pm 0.13$ \\
\hline & \multicolumn{6}{|c|}{$X_{\mathrm{CO}}=3 \times 10^{20} \mathrm{~cm}^{-2}\left(\mathrm{~K} \mathrm{~km} \mathrm{~s}^{-1}\right)^{-1}$} \\
\hline 0.0 & $0.97 \pm 0.02$ & $0.95 \pm 0.02$ & $0.96 \pm 0.02$ & $0.97 \pm 0.02$ & $0.94 \pm 0.03$ & $0.95 \pm 0.03$ \\
\hline 1.0 & $0.94 \pm 0.04$ & $0.92 \pm 0.04$ & $0.93 \pm 0.04$ & $0.92 \pm 0.05$ & $0.89 \pm 0.05$ & $0.91 \pm 0.05$ \\
\hline 2.0 & $0.89 \pm 0.05$ & $0.84 \pm 0.06$ & $0.86 \pm 0.06$ & $0.86 \pm 0.05$ & $0.81 \pm 0.07$ & $0.83 \pm 0.07$ \\
\hline 3.0 & $0.81 \pm 0.06$ & $0.79 \pm 0.07$ & $0.80 \pm 0.07$ & $0.77 \pm 0.07$ & $0.75 \pm 0.08$ & $0.76 \pm 0.07$ \\
\hline 4.0 & $0.81 \pm 0.06$ & $0.79 \pm 0.07$ & $0.80 \pm 0.07$ & $0.76 \pm 0.07$ & $0.74 \pm 0.08$ & $0.75 \pm 0.07$ \\
\hline 5.0 & $0.81 \pm 0.08$ & $0.78 \pm 0.08$ & $0.79 \pm 0.08$ & $0.74 \pm 0.09$ & $0.70 \pm 0.09$ & $0.72 \pm 0.09$ \\
\hline 6.0 & $0.70 \pm 0.09$ & $0.77 \pm 0.09$ & $0.74 \pm 0.10$ & $0.56 \pm 0.12$ & $0.66 \pm 0.11$ & $0.61 \pm 0.12$ \\
\hline 7.0 & $0.59 \pm 0.13$ & $0.64 \pm 0.16$ & $0.62 \pm 0.15$ & $0.38 \pm 0.14$ & $0.46 \pm 0.17$ & $0.42 \pm 0.16$ \\
\hline 8.0 & $0.56 \pm 0.17$ & $0.47 \pm 0.14$ & $0.53 \pm 0.17$ & $0.31 \pm 0.17$ & $0.18 \pm 0.10$ & $0.26 \pm 0.16$ \\
\hline
\end{tabular}

Lockman 1990; Nakanishi \& Sofue 2003, 2006; Kalberla et al. 2007). This is consistent with the results in Figure 5(b) given that our 0.6 resolution corresponds to $\sim 90 \mathrm{pc}$ at the Galactic center distance $(d=8.5 \mathrm{kpc})$.

The molecular gas is always the major phase $(\gtrsim 50 \%$ in mass) at the midplane, from the center to the Solar radius. The midplane of the gas disk moves slightly up and down locally (Sanders et al. 1984; Nakanishi \& Sofue 2003, 2006). By adopting the locations of the profile peaks as the approximate midplane, the $\mathrm{H}_{2}$ mass always dominates the $\mathrm{HI}$ mass in the inner MW; starting from $f_{\text {mol }} \sim 100 \%$ in the central region and becoming comparable to HI, $f_{\mathrm{mol}} \sim 50 \%$, around $|l|=60^{\circ}-75^{\circ}(R=7.4-8.2 \mathrm{kpc})$. Figure 4(b) also shows the dominance of the molecular gas in the midplane from the center to the solar radius. The dominance of $\mathrm{H}_{2}$ ends around $R \gtrsim 6 \mathrm{kpc}$, when the gas at high altitudes is included (see Section 4.2), since the HI gas becomes abundant at high altitudes.

\subsection{Radial Profile}

The Galactocentric distance appears to be the most important parameter for variations of $f_{\mathrm{mol}}$. Figure 4 shows the $f_{\mathrm{mol}}-R$ plots with the data integrated over (a) the whole disk thickness $|b|<30^{\circ}$ and (b) around the midplane $|b|<1$.5. In both cases, a radially decreasing trend is very clear. Figure 7 displays the northern and southern sides of the inner MW disk separately for $|b|<30^{\circ}$ and confirms that the radial dependence is the determinant. All of the $\mathrm{HI}$ and $\mathrm{H}_{2}$ gas within $|b|<30^{\circ}$ is integrated for these plots. The radial trend is also seen in the LVD (Figure 3(c)); declining from the innermost wedge at $R=0-1 \mathrm{kpc}$ to the outermost one.

The radial decrease and consistency between the northern and southern disks are clearer in Figure 7(c). The figure shows the average and rms scatter in radial bins with a $1 \mathrm{kpc}$ width, except for the central bin with a $0.5 \mathrm{kpc}$ width. Quantitatively, $f_{\text {mol }}$ decreases from about $\sim 100 \%$ at the center, remaining at $\gtrsim 50 \%$ out to $R \sim 6 \mathrm{kpc}$, and decreasing to $\sim 10 \%-20 \%$ at $R \sim 8.5 \mathrm{kpc}$ when integrated over the whole disk thickness $|b|<30^{\circ}$. The largest difference between the two sides is only about $11 \%$ at $R=6 \mathrm{kpc}$, and hence azimuthal variations are small. Table 1 lists $f_{\text {mol }}$ as a function of the Galactocentric radius $R$.

Previous studies analyzed the molecular fraction in galaxies on an azimuthally averaged basis and found similar radial trends (Sanders et al. 1985; Young \& Scoville 1991; Honma et al. 1995; Sofue et al. 1995; Wong \& Blitz 2002). The dominance of the radial dependence over the azimuthal dependence in the MW became clear in Figure 4.

The MW has a bar structure with a half-length of $4.4 \pm 0.5 \mathrm{kpc}$ (Benjamin et al. 2005). Barred spiral galaxies often show bright $\mathrm{CO}$ emission along their bars and at the bar ends (e.g., Sheth et al. 2002). On the northern side (Figure 7(a)), the most prominent bump around $R \sim 4.5 \mathrm{kpc}$, that is, $\sim 20 \%$ enhancement, may correspond the bar end. A similar enhancement is seen at the southern side as well at a slightly smaller radius $R \sim 3.9 \mathrm{kpc}$ (Figure 7(b)). The corresponding features can be identified in the LVD (Figure 3(c)): around $(l, v) \sim(31,100)$ for the northern side and $(343,-75)$ for the southern side, although for the northern side the entire wedge at $R=4-5 \mathrm{kpc}$ has a higher $f_{\mathrm{mol}}$. If these bumps are due to the bar, then the enhancement of $f_{\text {mol }}$ is only about $20 \%$ there.

Spiral arms and non-circular motions cause secondary variations on the radial decrease and locally steepen the radial gradient around the tangent radii of the spiral arms (Section 3.3 and Appendix). The arrows in Figures 7(a) and (b) show the locations of the tangent radii, and the thick arrows indicate the ones associated with prominent stellar spiral arms. The radial profiles seem to be steepened around the radii of the ScutumCrux arm (south) and possibly the Sagittarius-Carina arm (north), although the global radial trend is still maintained. No stellar counterparts have been found for the Sagittarius-Carina 

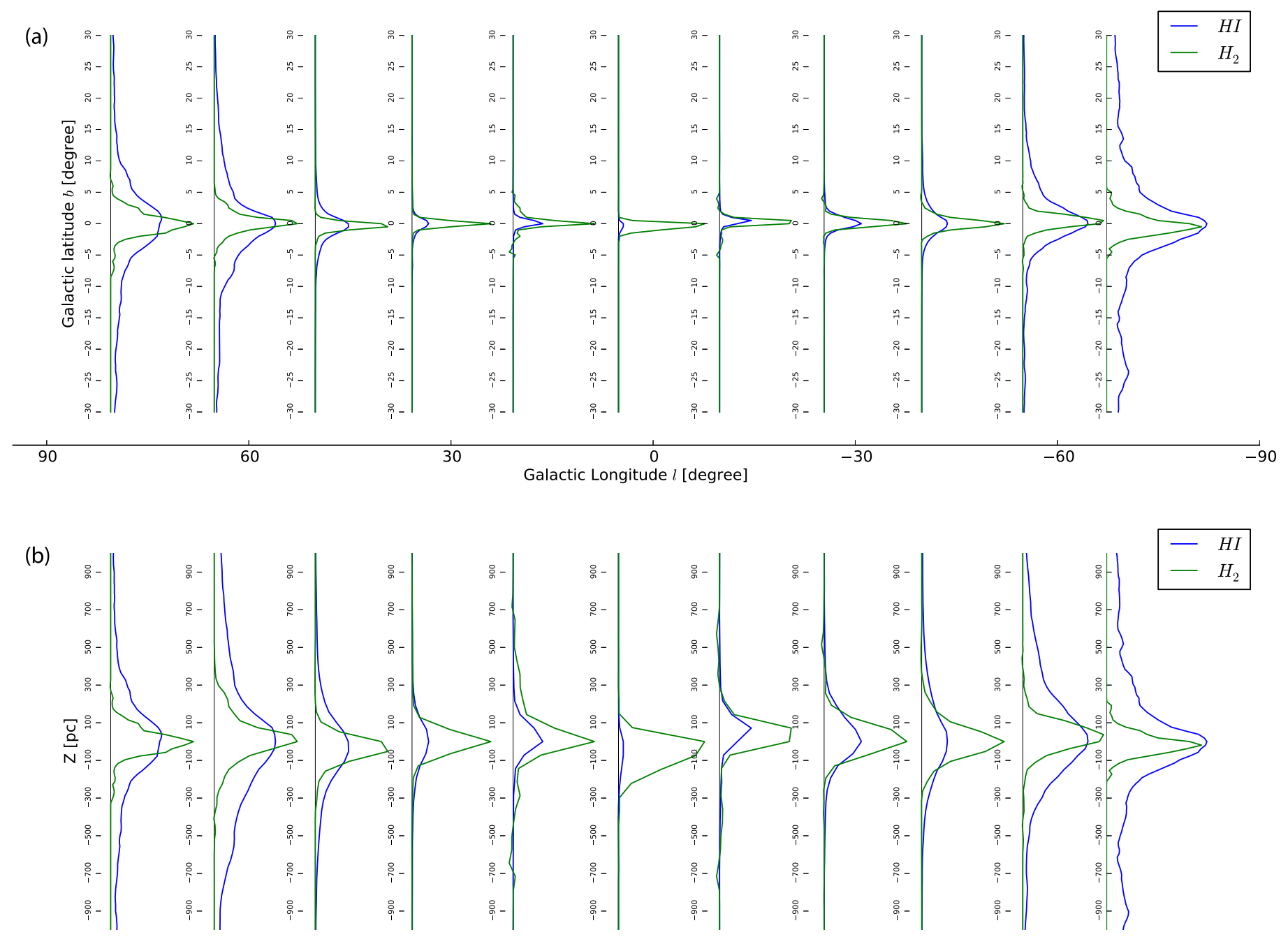

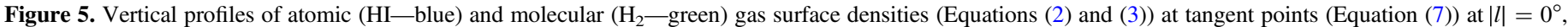

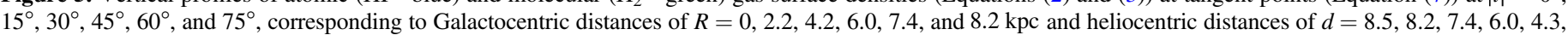

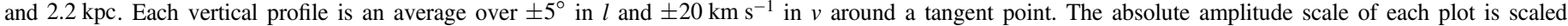

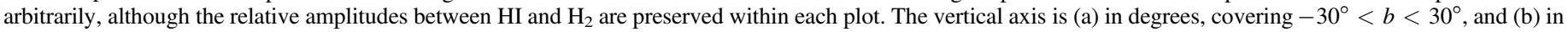
parsecs, covering -1000 to $1000 \mathrm{pc}$ in the direction of the disk thickness.

spiral arm (Drimmel \& Spergel 2001; Benjamin et al. 2005; Churchwell et al. 2009). Non-circular gas motions around this arm may be smaller as they are a response to a stellar spiral potential.

\subsection{Azimuthal Variations}

Azimuthal variations in $f_{\text {mol }}$ appear as scatter in Figures 4 and 7(a), (b) and are secondary compared to the dominant radial trend. The scatter at each radius in these figures represents variations of $f_{\mathrm{mol}}$ along the ring at that Galactocentric radius, thereby showing the range of azimuthal variation. This measurement is insensitive to the exact locations of the spiral arms, and therefore is robust. In Section 3.3 and the Appendix, we demonstrate that an LVD samples both the spiral arm and interarm regions. Even though the exact locations of the arms are debatable, it is certain that some points in Figure 7 should represent spiral arms while others show interarm regions. Thus, the scatter indicates the amount of arm/interarm variation. From Figure 7(c), the rms scatters are very small: $\sim 3 \%-12 \%$ at $R<6 \mathrm{kpc}$ and $7 \%-16 \%$ outward. In what follows, we discuss the reasons for the conclusion that the azimuthal variations are only $\sim 20 \%$ in the moleculedominated inner MW ( $R \lesssim 6 \mathrm{kpc} ; f_{\mathrm{mol}}>50 \%$ on average), while they increase to $\sim 40 \%-50 \%$ in the atom-dominated outskirts $\left(R \gtrsim 6 \mathrm{kpc} ; f_{\text {mol }}<50 \%\right)$.

Although the scatter plots (Figure 7) show the peak-to-peak variation for a given $R$, the measurement of the average azimuthal variation requires some interpretation. Figure 7 superposes the data from the bar, spiral arms, and interarm regions, all of which have their own deviations from the circular rotation which smear the plots in the horizontal direction (Section 3.3 and Appendix). Obviously, there must be very localized small regions where stellar feedback dissociates molecules into atoms. Thus, a peak-to-peak variation at each radius will hide the global trend of ISM phase change. In addition, the velocity dispersion of $\mathrm{HI}$ gas, $9-10 \mathrm{~km} \mathrm{~s}^{-1}$ (Malhotra 1995), is larger than that of $\mathrm{H}_{2}$ gas, $3-4 \mathrm{~km} \mathrm{~s}^{-1}$ 

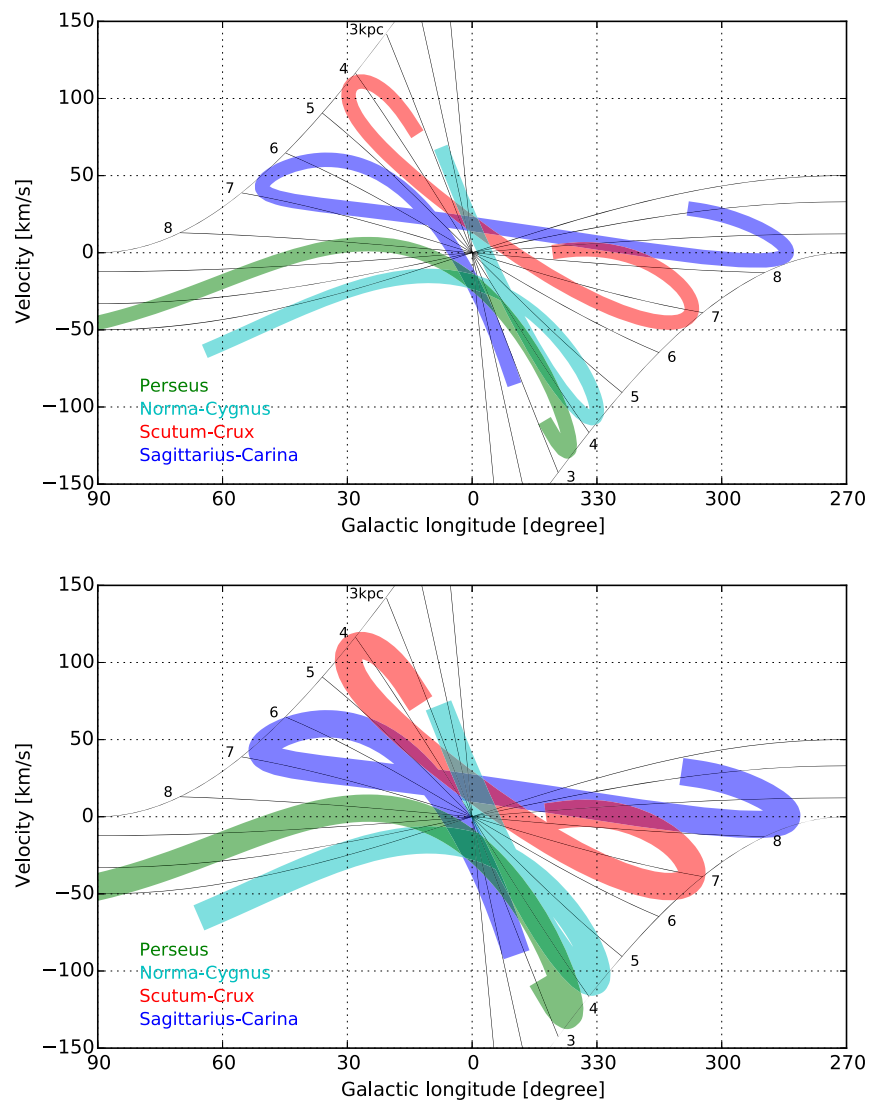

Figure 6. Model spiral arms on an LVD. Non-circular motions and velocity widths (due to increased velocity dispersions) in spiral arms are included as discussed in Section 3.3 and Appendix A. Only the Perseus and Scutum-Crux arms have corresponding stellar spiral arms. The Norma-Cygnus and Sagittarius-Carina arms are only seen in the ISM distribution, but not as stellar spiral arms, and might be interarm gas structures between the two stellar spiral arms. Spiral arms with (a) velocity widths that correspond roughly to the observed ones (about $\sim 10 \mathrm{~km} \mathrm{~s}^{-1}$ when the arms are seen perpendicular to our line of sight and $\sim 20-30 \mathrm{~km} \mathrm{~s}^{-1}$ around their tangent positions), (b) the maximum possible velocity widths $\left(\sim 20 \mathrm{~km} \mathrm{~s}^{-1}\right.$ when perpendicular to the line of sight, and up to $\sim 50 \mathrm{~km} \mathrm{~s}^{-1}$ at the tangent positions).

(cloud-cloud motions; Clemens 1985). Therefore, the HI emission is more smoothed out in the velocity direction; any enhancement of $\mathrm{HI}$ emission along the spiral arms, if it exists, is smoothed out in the interarm regions. This would increase the apparent $f_{\text {mol }}$ in the spiral arms and decrease it in the interarm regions. Even with these contaminations, the radial decrease is very clear, indicating that $R$ is the determinant parameter and that the other local variations are only secondary. From visual inspection of the vertical widths in Figure 7 with these contaminants in mind, we estimate $20 \%$ as the azimuthal, arm/interarm variations of $f_{\mathrm{mol}}$ at $R \lesssim 6 \mathrm{kpc}$ and $\sim 40 \%-50 \%$ at the outskirts, where $f_{\text {mol }}<50 \%$.

These arm/interarm variations in the inner MW $(R \lesssim 6 \mathrm{kpc})$ are much smaller than those from the complete phase transition suggested by the classic picture of ISM evolution. The gas remains molecular from interarm regions into spiral arms, and out again to the next interarm regions.

On the other hand, a rapid phase transition occurs in the outskirts $(R \gtrsim 6 \mathrm{kpc})$. For example, the northern side (Figure 7(a)) shows a few enhancements of $f_{\text {mol }}$ around $R \sim 6.0-6.5,6.5-7.2$, and 7.6-8.0 kpc. We can find corresponding enhancements of $f_{\mathrm{mol}}$ in the LVD (Figure 3(c)) as two clumps at $(l, v) \sim(35,45)$ and $(40,33)$ and a stretch around
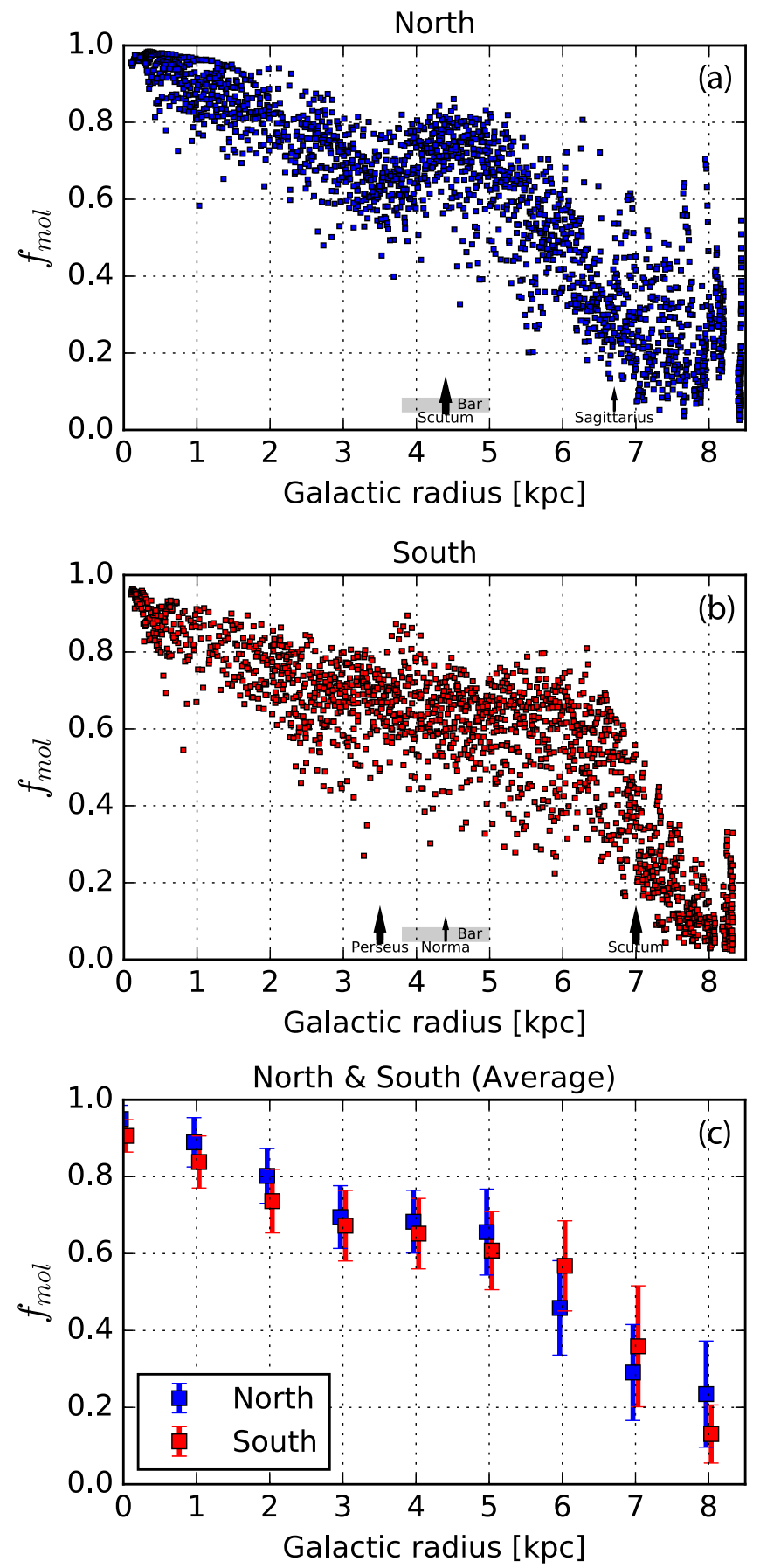

Figure 7. Same as Figure 4(a) (integrated over the whole disk thickness $|b|<30^{\circ}$ ), but (a) for the northern part of the Galactic plane, (b) for the southern part, and (c) showing averages and standard deviations in $1 \mathrm{kpc}$ bins for the northern and southern parts. (The points are slightly shifted in the horizontal direction to reduce overlaps.) The tangent points of the four spiral arms are indicated by arrows; the thick arrows indicate the two arms identified as stellar enhancements (i.e., potential). The bar end, $4.4 \pm 0.5 \mathrm{kpc}$, is indicated with a gray horizontal line. Only the tangent points of the spiral arms are indicated, but the data from other parts of the arms are distributed over all radii.

(22-32, 8), respectively. These appear even more distinct in the $\mathrm{CO}$ distribution (Figure 3(b)), and therefore are locally dense regions. Using Figure 6 as a reference for the qualitative locations of the spiral arms, the first two are probably on the Sagittarius-Carina arm. The third is along the Perseus arm. In 
the outskirts of the MW $(R \gtrsim 6 \mathrm{kpc})$ where the gas is predominantly atomic, $f_{\text {mol }}$ increases by $\sim 40 \%-50 \%$ in the spiral arm when the gas density is locally high. This is consistent with the absence of interarm MCs in the outer Galaxy where the ambiguity of heliocentric distance is not an issue (Heyer \& Terebey 1998; Heyer et al. 1998).

\subsection{Comparisons with Previous Studies}

Radial profiles of the $\mathrm{HI}$ and $\mathrm{H}_{2}$ surface densities, $\Sigma_{\mathrm{HI}}$ and $\Sigma_{\mathrm{H}_{2}}$, have been derived in previous studies (see reviews, Kalberla \& Kerp 2009; Heyer \& Dame 2015). Their measurements in the inner MW are uncertain due to the ambiguity problem caused by kinematic distance. Such errors can dilute azimuthal structures, such as spiral arms, as well as the radial profiles (Sections 1.1 and 3.2). Figure 8 demonstrates this problem and shows the profiles from the literature. For example, Nakanishi \& Sofue (2003) and Kalberla \& Dedes (2008) derived $\Sigma_{\mathrm{HI}}$ from the same HI data, but their results deviate from each other by about an order of magnitude (e.g., at $R=5-6 \mathrm{kpc}$ ). Nakanishi \& Sofue (2016) recently revised their analysis, but still the discrepancy remains large. Their total HI mass within the solar circle is smaller by a factor of $>2$ than that of Kalberla \& Dedes (2008). Nakanishi \& Sofue (2016) suggested that the discrepancy arises mainly from the adopted vertical profile models. To separate the gas at the near and far sides, these studies fit double Gaussian, $\operatorname{sech}^{2}$, or more detailed model profiles to emission profiles along $b$. The high-altitude wings of the model profiles may build up and introduce the large discrepancy in $\Sigma_{\mathrm{HI}}$.

The calculations of the $\Sigma_{\mathrm{HI}}$ and $\Sigma_{\mathrm{H}_{2}}$ radial profiles require heliocentric distances, and thus a resolution of the near/far ambiguity problem. Our analysis is less susceptible to this, as we do not derive $\Sigma_{\mathrm{HI}}$ and $\Sigma_{\mathrm{H}_{2}}$. Instead, we calculate $f_{\text {mol }}$, which is a distance-independent parameter in LVD and does not decompose the gas at the near and far sides. Figure 8(c) compares our $f_{\text {mol }}$ profile with those derived from some combinations of the previous $\Sigma_{\mathrm{HI}}, \Sigma_{\mathrm{H}_{2}}$ calculations. The radially declining trend is common, but the $f_{\text {mol }}$ value varies a lot due to the above difficulties. In this figure, Nakanishi \& Sofue (2016) is the closet to our result from the simpler method. This might indicate that their $\Sigma_{\mathrm{HI}}$ and $\Sigma_{\mathrm{H}_{2}}$ calculations, or at least their ratios, are closest to reality.

\subsection{Notes on Potential Systematic Errors}

Potential systematic errors in an LVD analysis have been discussed (e.g., Burton et al. 1992; Binney \& Merrifield 1998). Our analysis does not depend on the heliocentric distance and is relatively immune to the near-far distance ambiguity/ degeneracy problem. Nevertheless, there are some potential systematic errors, most of which we have already discussed. Here, we re-summarize them in terms of three error sources: (1) the overlap of the near and far sides in our analysis, (2) the potential difference in the motions of the $\mathrm{HI}$ and $\mathrm{H}_{2}$ components, and (3) the possible dark $\mathrm{HI}$ and $\mathrm{H}_{2}$ components.

The near-far distance degeneracy could indirectly affect our analysis. The near and far sides for a given $(l, v)$ are at the same $R$, and thus we analyzed them together. This treatment may occasionally mix a spiral arm and interarm region at the near and far sides (Section 3); in such a case, $f_{\text {mol }}$ likely represents the value in the spiral arm as the emission is typically brighter there. In addition, the fixed beam size and angular scale height
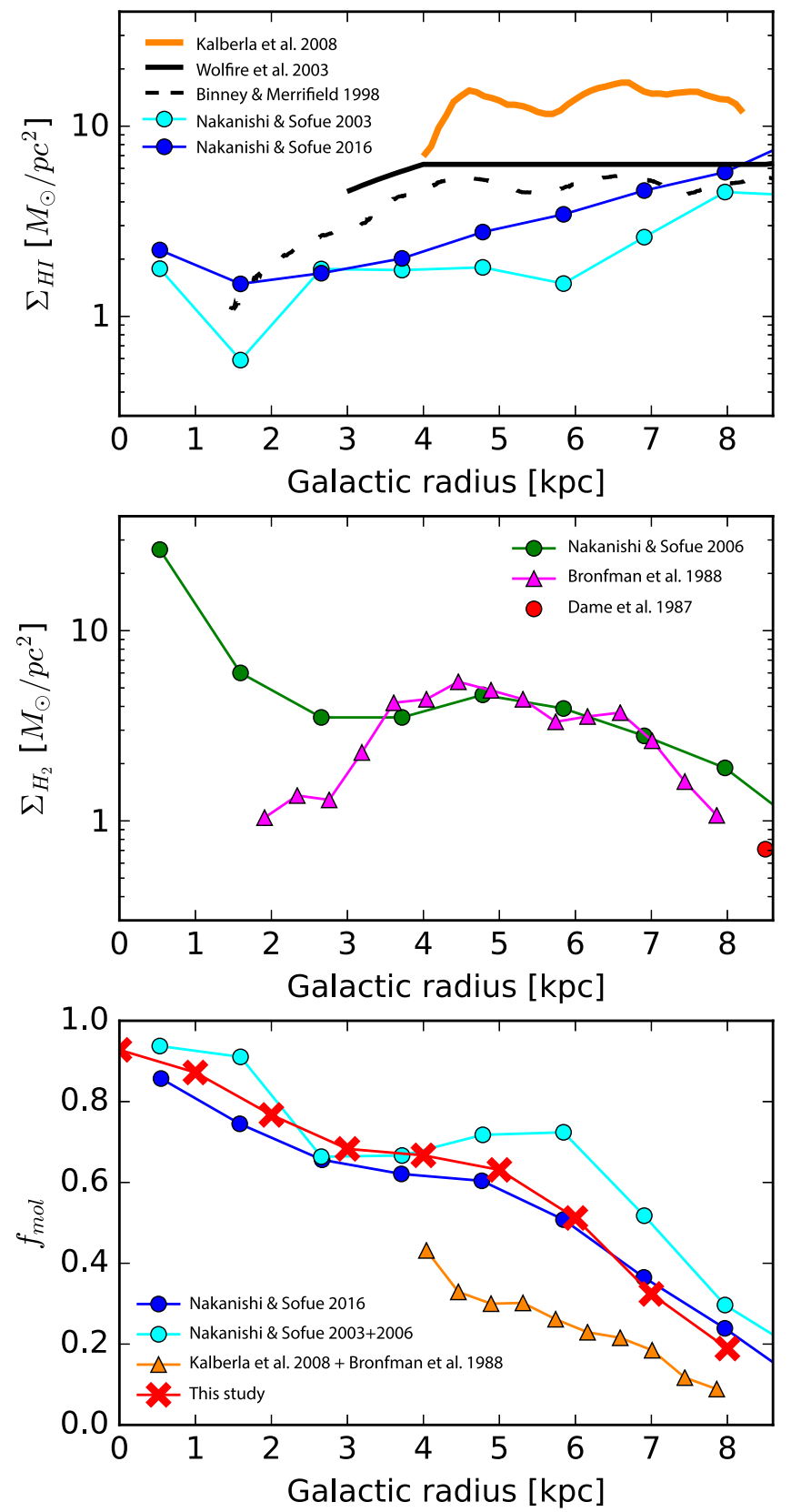

Figure 8. Radial profiles of (a) $\Sigma_{\mathrm{HI}}$, (b) $\Sigma_{\mathrm{H}_{2}}$, and (c) $f_{\text {mol }}$ derived from the literature for comparison. The $\Sigma_{\mathrm{HI}}$ profiles are from Kalberla \& Dedes (2008), Wolfire et al. (2003), Binney \& Merrifield (1998), and Nakanishi \& Sofue (2003, 2016). The data for the first three references are taken from Figure 3 of Kalberla \& Dedes (2008). The $\Sigma_{\mathrm{H}_{2}}$ profiles are originally from Nakanishi \& Sofue (2006), Bronfman et al. (1988), and Dame et al. (1987), and the data are taken from Heyer \& Dame (2015). $f_{\text {mol }}$ is calculated using the $\Sigma_{\mathrm{HI}}$ and $\Sigma_{\mathrm{H}_{2}}$ radial profiles, except in Nakanishi \& Sofue $\left(2016\right.$, which calculated $f_{\text {mol }}$ ) and this study. All of these studies, except for this study, attempted to resolve the ambiguity problem of kinematic distance, and thus suffer from uncertainties from the problem.

over which the emission is averaged correspond to different physical sizes between the near and far sides, and may dilute $f_{\text {mol }}$. This would likely result in increased scatter, particularly near the Sun $(R \sim 8.5 \mathrm{kpc})$, since a smaller physical size on the near side may pick up local variations, e.g., inside and outside MCs. Our averaging scale is large in $b$ (over $60^{\circ}$ or $3^{\circ}$ ) but relatively small in $l\left(0^{\circ} .6\right)$. In Figures 4 and 7 , some of the scatter around $R \sim 8.5 \mathrm{kpc}$ may come from this error. 
A difference in the motions of the $\mathrm{HI}$ and $\mathrm{H}_{2}$ (CO) components could cause an additional error. Most likely, the $\mathrm{HI}$ gas has a larger velocity width than $\mathrm{H}_{2}$, which would smear HI spiral arms and leak HI emission from arms into interarm regions. (The leak from interarms to arms should be smaller because the emission is concentrated in the arms more than in the interarms.) This would apparently raise $f_{\text {mol }}$ in the spiral arms and lower it in the interarm regions, possibly increasing the apparent arm-to-interarm variations (Section 4.3). For example, the turbulent velocity dispersion is larger for HI than for $\mathrm{H}_{2}$. If there are gradients in the rotation velocity versus the height (presumably only in an HI layer with a much larger scale hight), it would also increase the effective velocity width of $\mathrm{HI}$ in LVD.

We assumed optically thin $\mathrm{HI} 21 \mathrm{~cm}$ emission and a CO-to$\mathrm{H}_{2}$ conversion factor for calculations of the $\mathrm{HI}$ and $\mathrm{H}_{2}$ surface densities. If optically thick $\mathrm{HI}$ and $\mathrm{CO}$-dark $\mathrm{H}_{2}$ exist, then the $\mathrm{HI}$ and $\mathrm{CO}$ emission might not accurately trace the gas surface densities (Section 3.1.1). These dark $\mathrm{HI}$ and $\mathrm{H}_{2}$ should, to an extent, compensate for each other in the $f_{\text {mol }}$ calculation.

\section{DISCUSSION}

\subsection{ISM Evolution in Galaxies}

The azimuthal variation of the ISM phase is an important clue for characterizing the ISM evolution and star formation in galaxies. In Section 4, we demonstrated that in the MW, the azimuthal variations of the molecular fraction are much smaller than the radial variations. In the molecule-dominated inner disk $\left(f_{\mathrm{mol}}>50 \% ; R \lesssim 6 \mathrm{kpc}\right)$, the gas remains molecular in both the spiral arm and interarm regions. The azimuthal, arm/ interarm, variations in $f_{\text {mol }}$ are only about $20 \%$. In the atomdominated outskirts $\left(f_{\text {mol }}<50 \% ; R \gtrsim 6 \mathrm{kpc}\right)$, the variations can reach as high as $40 \%-50 \%$ in the spiral arms. The classification of "on-average" molecule-dominated and atomdominated regions is the key to understanding the discrepancies in the GMC evolution and lifetimes in the literature (Section 1.1; e.g., Scoville \& Hersh 1979; Blitz \& Shu 1980; Cohen et al. 1980; Sanders et al. 1985, and see also Koda 2013).

In the molecule-dominated disk of M51, the most massive MCs appear exclusively along spiral arms, while smaller MCs and unresolved molecular emission still dominate over HI in the interarm regions (Koda et al. 2009; Colombo et al. 2014). The majority of the unresolved emission needs to be in smaller MCs, since self-shielding is crucial for the survival of molecules in the interstellar radiation field (van Dishoeck \& Black 1988). These considerations suggest the coagulation and fragmentation of molecular gas structures in the spiral arms, rather than cycling between $\mathrm{HI}$ and $\mathrm{H}_{2}$ gas phases. The massive MCs and their $\mathrm{H}_{2}$ molecules are not fully dissociated into atomic gas, but are fragmented into smaller MCs upon leaving the spiral arms. The remnants of the fragmented massive MCs are detected in the interarm regions as smaller MCs (Koda et al. 2009). Dynamical stirring, spiral arm orbit crowding, as well as spiral arm shears likely play major roles in the ISM evolution in the molecule-dominated region. A similar difference in MC mass between spiral arms and interarm regions is found in the inner MW disk (Koda et al. 2006). The small azimuthal variations of $f_{\text {mol }}$ suggest that the evolution of the ISM and MCs in the inner MW are similar to the dynamically driven evolution in M51.
The LMC and M33 are rich in atomic gas, having fewer MCs than the MW and M51 (Engargiola et al. 2003; Fukui et al. 2009). Virtually all of the MCs there are associated with HI spiral arms and filaments. Molecular emission is absent in the interarm regions. This distribution indicates a short lifetime for MCs (i.e., of the order of an arm crossing timescale 30 Myr). Kawamura et al. (2009) found a similar lifetime of 20-30 Myr in the LMC by analyzing the fractions of MCs with and without associated star clusters and by translating them into the MC lifetime using cluster ages as a normalization. Miura et al. (2012) also found a similar lifetime of 20-40 Myr in M33. These short lifetimes appear to be common in the atomdominated galaxies. This is consistent with our results for the short lifetime of molecules in the atom-dominated outskirts of the MW (see also Heyer \& Terebey 1998). A similar transition is seen in M51, whose disk is largely molecule-dominated but becomes atom-dominated at the very outskirts (Koda et al. 2009).

A transitional case is found in the central $R \sim 2 \mathrm{kpc}$ region of M33 (Tosaki et al. 2011). The MC distribution is decoupled from the HI structures in the central region (Tosaki et al. 2011), while it coincides with the $\mathrm{HI}$ in the atom-dominated outer part (Engargiola et al. 2003). These decoupled MCs may be entities surviving for long times, greater than a galactic rotation period, during which the HI structures would be smeared out. $f_{\text {mol }}$ increases to $\gtrsim 50 \%$ toward the center from $\sim 0 \%-20 \%$ in the outskirts (Tosaki et al. 2011, see their Figure 4).

All of the above point to an integrated view of ISM evolution in galaxies. In the inner parts of galaxies where the molecular gas is overall dominant, the gas remains molecular even in the interarm regions. On the other hand, in the outer atomdominated regions, the phase transition occurs in the gas, becoming molecular as it enters spiral arms but being photodissociated back into the atomic phase upon exit. Figure 9 presents a schematic illustration of the ISM evolution in the inner and outer disk.

\subsection{The Azimuthal Constancy and Radial Gradient}

$f_{\text {mol }}$ decreases monotonically with Galactic radius, while its azimuthal variation is small $\sim 20 \%$. This suggests that the gasphase balance is approximately in equilibrium at a given radius, and that the gas cycling between $\mathrm{HI}$ and $\mathrm{H}_{2}$ is in a steady state in the azimuthal direction. The parameter that controls the phase balance exhibits a strong variation with the radius. Here, we show that a simple model can explain the observed radial trend. This model is based on one principle and two assumptions.

First, if the ISM continuously cycles the gas between the HI and $\mathrm{H}_{2}$ phases, then the steady state suggests that the $\mathrm{HI} \rightarrow \mathrm{H}_{2}$ mass conversion rate " $M_{\mathrm{HI}} / \tau_{\mathrm{HI}}$ " is equal to that for $\mathrm{H}_{2} \rightarrow \mathrm{HI}$ " $M_{\mathrm{H}_{2}} / \tau_{\mathrm{H}_{2}}$ " at each radius (i.e., the continuity principle; Scoville \& Hersh 1979). Therefore, we have

$$
\frac{M_{\mathrm{H}_{2}}}{M_{\mathrm{HI}}}=\frac{\tau_{\mathrm{H}_{2}}}{\tau_{\mathrm{HI}}} .
$$

Based on observations, the mass in the $\mathrm{H}$ II phase is taken to be negligible, and the fraction of gas converted to stars per orbit is small (Bigiel et al. 2008).

Second, we assume that molecules and MCs form from HI gas exclusively in spiral arms (or in the bar). Hence, the $\mathrm{HI} \rightarrow \mathrm{H}_{2}$ conversion timescale-or, equivalently, the lifetime of a typical $\mathrm{HI}$ atom $\left(\tau_{\mathrm{HI}}\right)$ - scales with the arm-to-arm travel 


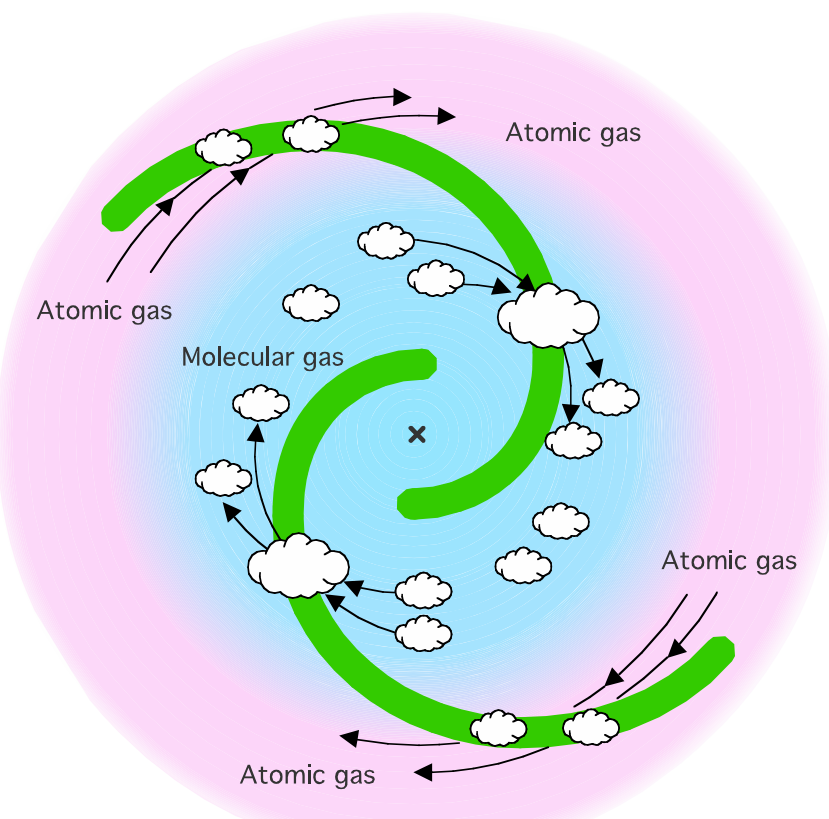

Figure 9. Schematic illustration of ISM evolution in the MW. In the moleculedominated inner disk (light blue), molecular clouds coagulate into more massive molecular clouds and then fragment into smaller ones during spiral arm passage. The gas stays in molecular clouds even in the interarm regions. On the other hand, in the atom-dominated outer disk (pink), the gas-phase transition occurs: the gas becoming molecular as it enters spiral arms, but being dissociated back into the atomic phase upon exit. Similar azimuthal evolution is observed in the atom-dominated disks of the LMC and M33, and in the molecule-dominated disk of M51. (Note that the radial range of this illustration is assumed to be within the co-rotation radius of the MW.)

time. For a spiral galaxy like the MW,

$$
\tau_{\mathrm{HI}}=\frac{2 \pi}{\epsilon_{\mathrm{HI}} m\left(\Omega(R)-\Omega_{\mathrm{p}}\right)},
$$

where $m$ is the number of spiral arms, $\epsilon_{\mathrm{HI}}$ is the $\mathrm{HI} \rightarrow \mathrm{H}_{2}$ conversion efficiency in a single arm encounter, $\Omega_{\mathrm{p}}$ is the constant pattern speed of the spiral arms, and $\Omega(R)=V_{0} / R$ is the angular speed of gas for a flat rotation curve. $\epsilon_{\mathrm{HI}}$ may depend on the spiral arm strength and HI density, e.g., if gravitational collapse followed by spiral arm compression is necessary for the conversion, but we take it to be constant within $R<R_{0}$ since the HI density does not vary much within the solar circle (Burton \& Gordon 1978; Scoville \& Sanders 1987; Nakanishi \& Sofue 2003). (Note that beyond the co-rotation radius $R_{\mathrm{cr}} \equiv V_{0} / \Omega_{\mathrm{p}}$, the sign of Equation (10) should be flipped.)

Third, we assume that the $\mathrm{H}_{2} \rightarrow \mathrm{HI}$ conversion timescalethe lifetime of a typical $\mathrm{H}_{2}$ molecule $\left(\tau_{\mathrm{H}_{2}}\right)$-is constant. This is justified if the dissociation of molecules and MCs is due to the internal physics of the MCs. For example, if many cycles of star formation are required to completely dissociate all of the molecules in an MC, then $\tau_{\mathrm{H}_{2}}$ could be constant in a statistical sense when averaged over the MC mass spectrum, although it may vary for individual clouds.

Equations (9) and (10) give

$$
\frac{M_{\mathrm{H}_{2}}}{M_{\mathrm{HI}}}=\frac{\tau_{\mathrm{H}_{2}} \epsilon_{\mathrm{HI}} m}{2 \pi}\left(\Omega(R)-\Omega_{\mathrm{p}}\right)
$$
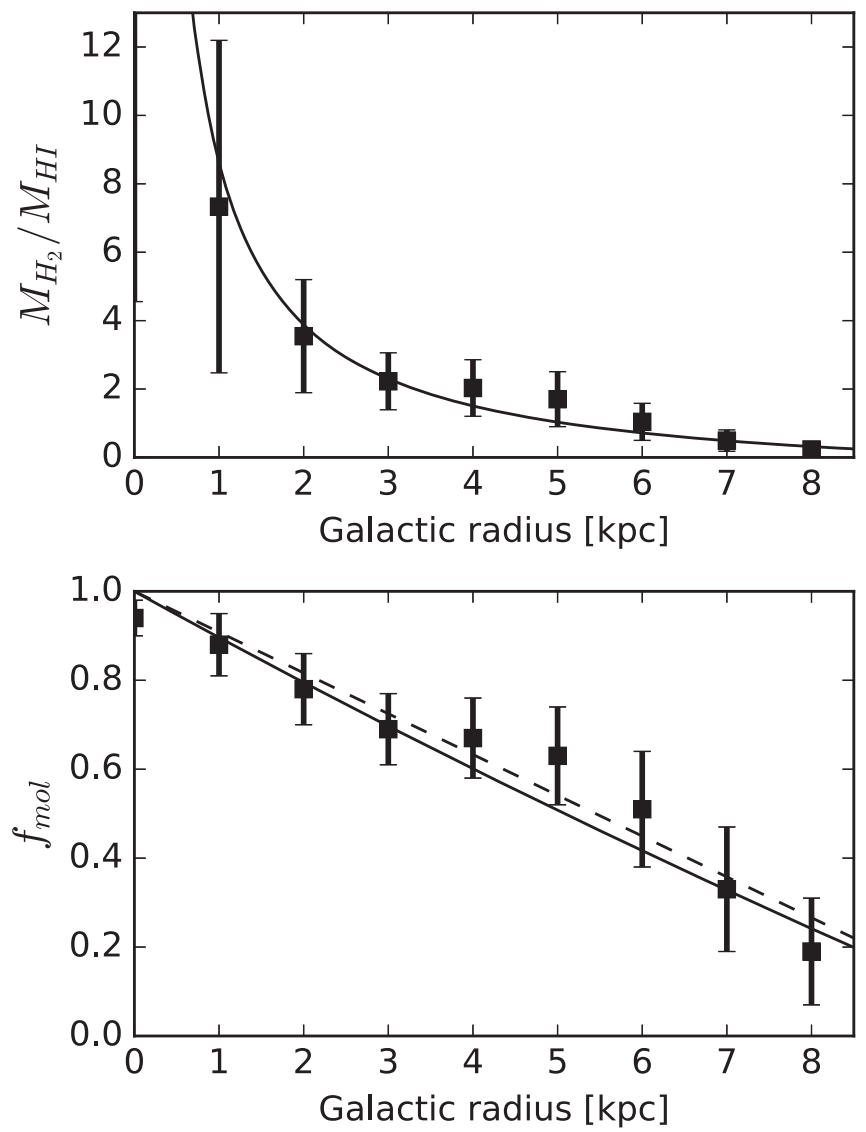

Figure 10. Least-square fits. The data are the averages of the northern and southern parts and from Table 1. (a) A fitting function of $M_{\mathrm{H}_{2}} / M_{\mathrm{HI}}=\alpha t-\beta$, where $t=1 / R$ is used. Note that $M_{\mathrm{H}_{2}} / M_{\mathrm{HI}}=f_{\mathrm{mol}} /\left(1-f_{\mathrm{mol}}\right)$. The fit provides $(\alpha, \beta)=(9.5 \pm 0.5,0.87 \pm 0.23)$ and the solid line. (b) The same data and fitted line as in (a), but converted to the $f_{\mathrm{mol}}-R$ plot. Another fit is also made using a simple linear function, $f_{\mathrm{mol}}=1-\gamma R$. The $y$-intercept is around 1 in the data, so we fix it to 1 . The fit provides $\gamma=0.089 \pm 0.052$ and is presented by the dashed line.

$$
=\frac{\tau_{\mathrm{H}_{2}} \epsilon_{\mathrm{HI}} m \Omega_{\mathrm{p}}}{2 \pi}\left(\frac{R_{\mathrm{cr}}}{R}-1\right) .
$$

This model predicts that the molecular fraction decreases with radius. Qualitatively, $\tau_{\mathrm{HI}}(\propto$ the arm-to-arm travel time) increases with increasing Galactic radius, while $\tau_{\mathrm{H}_{2}}$ is set to be constant, thus naturally explaining the transition smoothly from the molecule-dominated inner part to the atom-dominated outer part.

For quantitative assessment, we make a fit to the data (Table 1) using a fitting function with the form of Equation (12), $M_{\mathrm{H}_{2}} / M_{\mathrm{HI}}=\alpha t-\beta$, where $t \equiv 1 / R, \beta \equiv \tau_{\mathrm{H}_{2}} \epsilon_{\mathrm{HI}} m \Omega_{\mathrm{p}} / 2 \pi$, and $\alpha / \beta \equiv R_{\mathrm{cr}}$. We do not convert this equation to that for $f_{\mathrm{mol}}$ since it is not as simple for fitting purposes. The data point at $R=0 \quad\left(M_{\mathrm{H}_{2}} / M_{\mathrm{HI}}=12.9 \pm 8.5\right)$ is excluded from the fit because the ratio diverges there (Equation (12)). The fit results in $(\alpha, \beta)=(0.95 \pm 0.5,0.87 \pm 0.23)$. Figure 10 shows the result of the fit in panel (a) and its conversion to $f_{\text {mol }}$ in panel (b). Clearly, Equation (12) reproduces the observed radial trend well.

This result translates to $R_{\mathrm{cr}}=10.9 \pm 2.9 \mathrm{kpc}, \Omega_{\mathrm{p}}=V_{0} / R_{\mathrm{cr}}=$ $20.1 \pm 5.3 \mathrm{~km} \mathrm{~s}^{-1} \mathrm{kpc}^{-1}$, and $\tau_{\mathrm{H}_{2}}=2 \pi \beta /\left(\epsilon_{\mathrm{HI}} m \Omega_{\mathrm{p}}\right)=(132 \pm$ 49) $\epsilon_{\mathrm{HI}}^{-1}(2 / m)$ Myr. These are consistent with those $\left(\Omega_{\mathrm{p}}\right.$, $\left.R_{\mathrm{cr}}\right) \sim\left(18.4 \mathrm{~km} \mathrm{~s}^{-1} \mathrm{kpc}^{-1}, 11.9 \mathrm{kpc}\right)$ derived, e.g., by Bissantz 
Table 2

Parameters From Fit

\begin{tabular}{lccc}
\hline \hline & & \multicolumn{2}{c}{$X_{\mathrm{CO}}\left(\mathrm{cm}^{-2}\left(\mathrm{~K} \mathrm{~km} \mathrm{~s}^{-1}\right)^{-1}\right)$} \\
\cline { 3 - 4 } Parameter & Unit & $2 \times 10^{20}$ & $3 \times 10^{20}$ \\
\hline$\alpha$ & & $9.5 \pm 0.5$ & $14.3 \pm 0.8$ \\
$\beta$ & & $0.87 \pm 0.23$ & $1.31 \pm 0.34$ \\
$R_{\mathrm{cr}}(=\alpha / \beta)$ & $\mathrm{kpc}$ & $10.9 \pm 2.9$ & $10.9 \pm 2.9$ \\
$\Omega_{\mathrm{p}}\left(=V_{0} / R_{\mathrm{cr}}\right)$ & $\mathrm{km} \mathrm{s}^{-1} \mathrm{kpc}^{-1}$ & $20.1 \pm 5.3^{\mathrm{a}}$ & $20.2 \pm 5.3^{\mathrm{a}}$ \\
$\tau_{\mathrm{H}_{2}}\left(=2 \pi \beta / m \epsilon_{\mathrm{HI}} \Omega_{\mathrm{p}}\right)$ & $\mathrm{Myr}$ & $331 \pm 123^{\mathrm{b}}$ & $499 \pm 184^{\mathrm{b}}$ \\
\hline
\end{tabular}

Notes.

assumed $V_{0}=220 \mathrm{~km} \mathrm{~s}^{-1}$

${ }^{\mathrm{b}}$ Assumed $\epsilon_{\mathrm{HI}}=0.4$ and $m=2$.

et al. (2003) after correction for the adopted $R_{0}$, although all of the measurements in the literature have considerable uncertainties. The MW has two stellar spiral arms, and therefore $m=2$. We estimate $\epsilon_{\mathrm{HI}} \sim 0.4$ by translating the $\sim 20 \%$ azimuthal variation of $f_{\text {mol }}$ where the $\mathrm{HI}$ fraction is about $50 \%$ $\left(f_{\text {mol }}=0.5\right.$; Section 4.3$)$. Hence, $\tau_{\mathrm{H}_{2}} \sim 330 \mathrm{Myr}$ on average within the solar circle. Table 2 summarizes the derived parameters.

$\tau_{\mathrm{HI}}$ is roughly comparable to the gas rotation timescale with respect to the spiral pattern when $\epsilon_{\mathrm{HI}} m \sim 1$ (Equation (10)) as in our case, and $\tau_{\mathrm{HI}} \sim 40,140,320,670$, and $1700 \mathrm{Myr}$ at $R=1,3,5,7$, and $9 \mathrm{kpc}$, respectively. The lifetime of $\mathrm{H}_{2}$ is longer than the rotation timescale in the inner MW, and the gas remains mostly molecular during the arm-to-arm travel time. (This is true even if the number of spiral arms is assumed to be $m=4: \tau_{\mathrm{H}_{2}}(\propto 1 / m)$ would be twice as short, but the arm-to-arm travel time is also twice as short.) The opposite is the case in the outskirts where molecules survive for only a small fraction of the rotation timescale and exist only around spiral arms. Indeed, there are MCs around spiral arms in the outer MW with $R>R_{0}$ (Heyer \& Terebey 1998; Heyer et al. 1998), but averaged along the annulus, $f_{\text {mol }} \ll 1$.

Some of the parameters may vary in other environments. For example, $\tau_{\mathrm{HI}}$ could be longer in the outer MW and in the atomdominated galaxies (e.g., the LMC and M33) because of an absence of (or weaker) stellar spiral structures (i.e., smaller $m$ and/or lower $\epsilon_{\mathrm{HI}}$ ), and because of the intrinsically low gas density (i.e., lower $\epsilon_{\mathrm{HI}}$-at a low density, spiral arm compression, when it exists, may not convert $\mathrm{HI}$ to $\mathrm{H}_{2}$ efficiently). $\tau_{\mathrm{H}_{2}}$ could be smaller if the average MC mass is lower. All of these keep $M_{\mathrm{H}_{2}}$ and $f_{\text {mol }}$ lower and qualitatively explain the HI-dominated regions.

We assumed that spiral arms or a bar are the trigger of the HI $\rightarrow \mathrm{H}_{2}$ conversion, however, this model works even if the conversion is due to another physical mechanism as long as the timescale is comparable/proportional to the rotation timescale at that radius. For example, if MCs form by the agglomeration of atomic clouds and if their collision timescale is set by their velocity difference due to differential galactic rotation, $\tau_{\mathrm{HI}}$ would have a similar dependence on $\Omega(R)$ (e.g., Scoville \& Hersh 1979; Wyse 1986; Wyse \& Silk 1989; Tan 2000). This model assumed that the arms/bar significantly enhance the HI $\rightarrow \mathrm{H}_{2}$ conversion. We should note that the conversion could occur at a much lower rate, e.g., in a dwarf galaxy without prominent spiral arms/bar, if there are local density fluctuations.

\subsection{Comments on the Midplane Pressure}

The radial decrease of $f_{\text {mol }}$ alone has been known for some time (Sanders et al. 1984; Young \& Scoville 1991; Honma et al. 1995; Sofue et al. 1995; Wong \& Blitz 2002), although the azimuthal variation was rarely analyzed. This radial trend is often discussed in relation to the hydrostatic pressure at the midplane of the galactic disk under the gas and stellar gravitational potentials, $P_{\text {mid }}$ (Wong \& Blitz 2002; Blitz \& Rosolowsky 2004, 2006; Field et al. 2011; Hughes et al. 2013; see also Elmegreen 1989, 1993). There is an empirical linear correlation between $P_{\text {mid }}$ and the mean ratio of molecular to atomic hydrogen $R_{\text {mol }}$. However, it remains unclear how $P_{\text {mid }}$ is physically coupled to the MCs, the major reservoir of molecular gas.

It is often mistakenly assumed that the ambient midplane pressure confines the gas in MCs. This is not the case, and the nature of the pressure needs to be considered carefully. The thermal and magnetic pressures are not strong enough to confine the gas within a MC, while the pressure from largescale turbulence is not a confining pressure. Adopting a supersonic dispersion of $3-5 \mathrm{~km} \mathrm{~s}^{-1}$, the internal turbulent pressure of MCs is $P_{\mathrm{MC}} / k \sim 10^{5-6} \mathrm{~K} \mathrm{~cm}^{-3}$. This exceeds the thermal pressure of the ambient gas, $P_{\mathrm{th}} / k \sim 10^{3-4} \mathrm{~K} \mathrm{~cm}^{-3}$, by 1-2 orders of magnitude. The magnetic pressure is also too low, $P_{\mathrm{B}} / k=B^{2} /(8 \pi k) \sim 10^{4} \mathrm{~K} \mathrm{~cm}^{-3}$, using the observed magnetic strength of $B<10 \mu \mathrm{G}$ in the ambient medium (Crutcher 2012). The external turbulent pressure $P_{\text {turb }}$ which supports the vertical structure does not confine the gas within MCs since it is mostly unisotropic/directional-an MC may feel ram pressure from the direction of its motion with respect to the ambient gas, but this head wind is only from one side of the MC and there is no turbulent pressure on its trailing side. The midplane pressure cannot directly confine gas in MCs.

A careful assessment of the causalities and physical mechanisms for the quasi-equilibrium of the gas phases is needed. Ostriker et al. (2010) made such an attempt and distinguished $P_{\text {th }}$ from $P_{\text {mid }}\left(=P_{\text {turb }}+P_{\text {th }}\right)$, but had to assume that the $P_{\text {mid }}$ from the vertical dynamical equilibrium and the $P_{\text {th }}$ for the thermal equilibrium are coupled (they assumed $\left.P_{\text {mid }} / P_{\text {th }}=5\right)$. The establishment of such energy partitionfrom the galaxy center to outskirts and between spiral arms and interarm regions - is the key to understanding the phase balance in the ISM. In addition to the complex energy balance in the ISM, many parameters have radial dependences and are inter-dependent. For example, $P_{\text {mid }}$ is often calculated from stellar and gas surface densities alone, and one should ask, e.g., which parameter really causes the phase balance (pressure or density?). Future studies should carefully sort out these degeneracies.

\section{CONCLUSIONS}

We analyzed the variations of the molecular fraction $f_{\mathrm{mol}}$ in the MW in the radial and azimuthal directions by using archival $\mathrm{CO}(J=1-0)$ and $\mathrm{HI} 21 \mathrm{~cm}$ emission data. $f_{\text {mol }}$ decreases monotonically from the globally molecule-dominated central region $\left(f_{\text {mol }} \sim 100 \%\right)$ to the mostly atom-dominated outer region of the MW $\left(f_{\text {mol }} \sim 10 \%-20 \%\right.$ at the Solar radius when integrated over the whole gas disk thickness $|b|<30^{\circ}$ and $\sim 50 \%$ at the disk midplane). The azimuthal variation, and hence the arm/interam variation, of the gas phase is small, $\sim 20 \%$, within the molecule-dominated inner disk $(R \lesssim 6 \mathrm{kpc}$; 
$f_{\text {mol }} \gtrsim 50 \%$ ). The gas remains largely molecular even after spiral arm passage and in interarm reigons. This is at variance with the classic scenario of ISM evolution for rapid and complete phase transitions during spiral arm passage. On the contrary, the rapid gas-phase change occurs only in the atomdominated outskirts $\left(R \gtrsim 6 \mathrm{kpc} ; f_{\mathrm{mol}} \lesssim 50 \%\right)$. The average $f_{\text {mol }}$ around the solar neighborhood is about $20 \%$ including the $\mathrm{HI}$ gas at high Galactic disk altitudes, while it is still $50 \%$ at the disk midplane at the solar radius. The gas remains largely molecular across the spiral arms and interam regions in its inner disk, while in the outskirts the molecular gas is localized in the spiral arms and becomes atomic in the interarm regions. This classification of average atom-dominated and moleculardominated regions appears to be applicable to other nearby galaxies, such as LMC, M33, and M51.

We also demonstrated that a simple model of the phase balance and mass continuity in the $\mathrm{HI}$ and $\mathrm{H}_{2}$ cycling can explain the observed radial trend if the $\mathrm{HI} \rightarrow \mathrm{H}_{2}$ conversion occurs on a galactic rotation timescale (e.g., due to spiral arm compressions) and the $\mathrm{H}_{2} \rightarrow \mathrm{HI}$ conversion has a constant timescale (e.g., due to internal physics of MCs, such as multiple cycle of star formation).

We thank Roberta Paladini, Tom Dame, Lena Murchikova, and Jim Barrett for useful discussions. We also thank the anonymous referee for careful reading. J.K. thanks the California Institute of Technology for hospitality during extended visits. This work is supported by the NSF through grant AST-1211680. J.K. also acknowledges the supports from NASA through grants NNX09AF40G, NNX14AF74G, a Herschel Space Observatory grant, and an Hubble Space Telescope grant.

\section{APPENDIX A MW SPIRAL ARMS IN AN LVD}

An LVD is a tool used to investigate the spiral arms and interarm regions in the MW. Here, we demonstrate how spiral arms appear in an LVD using an observationally motivated, but simplistic, logarithmic spiral model.

\section{A.1. Spiral Arms in the $M W$}

The MW is likely to have two major, and potentially two minor, spiral arms (Drimmel 2000; Benjamin 2008; Churchwell et al. 2009; Robitaille et al. 2012). Steiman-Cameron et al. (2010) obtained the geometry of the four spiral arms in the gas component using the longitude profile of [C II] $158 \mu \mathrm{m}$ line emission. They assumed that each emission peak indicates the longitude of a spiral arm tangent point, and that two tangential longitudes of a spiral arm determine the geometry of the arm. Figure 11(a) shows the face-on projection of the four gas arms. Only two of the four arms were identified in the stellar distributions (Drimmel 2000; Benjamin 2008; Churchwell et al. 2009). Robitaille et al. (2012) concluded that a model with two major and two minor spiral arms can reproduce the range of emission from stellar to dust infrared, and to polycyclic aromatic hydrocarbon (PAH) emission. The MW has a relatively large bar at the center (Benjamin et al. 2005), and external galaxies with such large bars most often have only two significant stellar spiral arms. At the same time, optical images of a number of barred galaxies, e.g., from the Hubble Space Telescope archive, show filamentary dust-extincted lanes between stellar spiral arms. These ISM concentrations would appear as apparent spiral arms if observed in tracers of the ISM and associated star formation in an LVD. As per historical convention, we call the four gas arms the Sagittarius-Carina, Scutum-Crux, Norma-Cygnus, and Perseus arms. The Perseus and Scutum-Crux arms are the stellar spiral arms (thick lines in Figure 11(a)).

\section{A.2. A Simple Model of Spiral Arms in A LVD}

These spiral arms are translated into the $l-v$ space using a model of MW rotation. Pineda et al. (2013) assumed a pure circular flat rotation curve with a velocity of $V_{0}=220 \mathrm{~km} \mathrm{~s}^{-1}$ and the Sun at a radius of $R_{0}=8.5 \mathrm{kpc}$. At a general location in the MW disk, its Galactic radius $R$ and longitude $l$ determine an observed line-of-sight velocity $v_{l}$ as

$$
v_{l}=V_{0}\left(\frac{R_{0}}{R}-1\right) \sin l,
$$

which is the same as in Equation (5). Figure 11(b) (dotted lines) shows the spiral arms with circular rotaiton on an LVD.

Non-circular motions, i.e., deviations from purely circular rotation, change the arm locations on the LVD. The gas and stars take elongated (oval) orbits due to the kinematic density wave (Onodera et al. 2004). They slow down and stay long around the apocenter, since it is the outermost radius of the orbit in the Galactic gravitational potential. This slow down causes an enhanced density in the spiral density wave. Koda \& Sofue (2006) demonstrated this density enhancement in the case of a bar potential, and the same mechanism should work in a spiral potential (see Onodera et al. 2004). Therefore, the rotation velocity $V_{r}$ with non-circular motions on a spiral arm (i.e., apocenter) should be smaller than that with purely circular rotation $V_{0}$. The direction of motion should also be tilted slightly inward toward the Galactic center, by a small angle $\alpha$, with respect to the tangential direction of the circular orbit. Figure 11(c) shows the definitions of $V_{r}$ and $\alpha$. In this case, the spiral arm is expressed as

$$
\begin{aligned}
v_{1}= & V_{r}\left[\left(\frac{R_{0}}{R}\right) \sin l \cos \alpha \pm \sqrt{1-\left(\frac{R_{0}}{R} \sin l\right)^{2} \sin \alpha}\right] \\
& -V_{0} \sin l
\end{aligned}
$$

The "+" is for the far side and "-_" for the near side since a line of sight typically passes a single spiral arm twice (see Section 3.2 for near/far distances). The Sun is assumed to be on a circular orbit. We should note that this expression is only for the points on the spiral arms, not for other parts of the orbit.

Figure 11(b) (solid lines) shows the spiral arms with the noncircular motions. We arbitrarily assumed a constant $\alpha=5^{\circ}$ and $V_{r}=215 \mathrm{~km} \mathrm{~s}^{-1}$ for all Galactocentric radii $R$. The arms form coherent loops as in Figure 11(b), and the interarm regions appear between the spiral arm loops. The purpose of this model is only the qualitative demonstration of the effects of noncircular motions around spiral arms on an LVD. $\alpha$ and $V_{r}$ should, of course, vary with radius and could be different between the spiral arms. Nevertheless, this model appears closer to the spiral arms traced by the distribution of $\mathrm{H}$ II regions in an LVD (Sanders et al. 1985) than does a model with only pure circular rotation. 

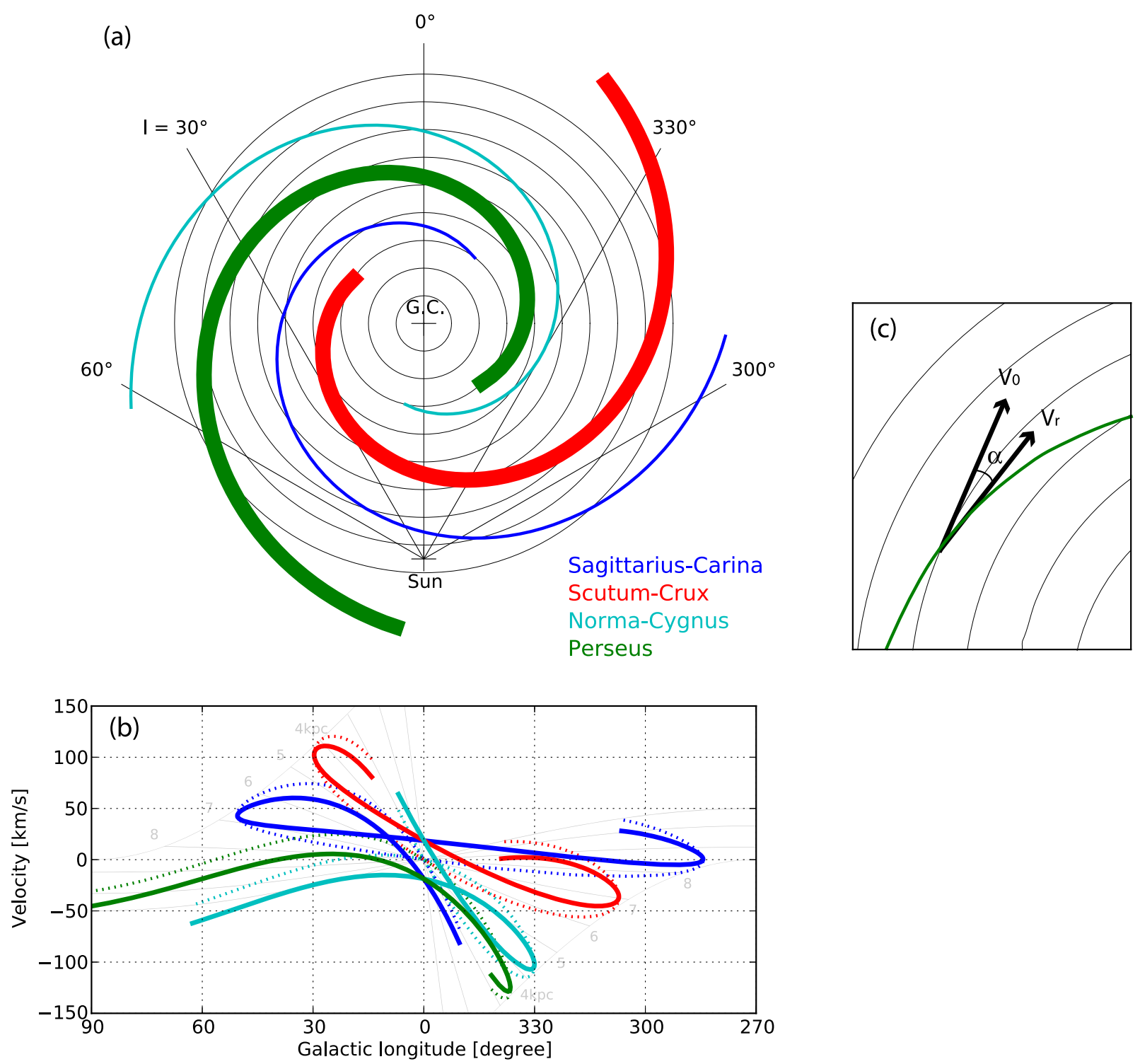

Figure 11. Logarithmic spiral arms of the Milky Way. (a) Logarithmic spiral arms from Churchwell et al. (2009), Steiman-Cameron et al. (2010). Four arms, Sagittarius-Carina (blue), Scutum-Crux (red), Norma-Cygnus (cyan), and Perseus arms (green), are often identified by studies with ISM and star formation tracers while only the Perseus and Scutum-Crux arms (thick lines) are identified as stellar spiral arms. Concentric circles around the Galactic Center (G.C.) are drawn at a $1 \mathrm{kpc}$ interval. (b) Longitude-velocity diagram of the four spiral arms. Dotted lines are for the model of pure circular rotation with a constant rotation velocity of $220 \mathrm{~km} \mathrm{~s}^{-1}$ and the solar Galactcentric radius of $R=8.5 \mathrm{kpc}$. Solid lines are for the model with non-circular motions, $\alpha=5^{\circ}$ and $V_{r}=215 \mathrm{~km} \mathrm{~s}{ }^{-1}$. (c) Explanation of the tilt angle $\alpha$ measured from the pure circular rotation vector $V_{0}$ to the non-circular motion vector $V_{r}$.

\section{A.3. Effects of Spiral Arms and Non-circular Motions in an $L V D$}

This toy model provides insight into how spiral arms and interarm regions should appear in an LVD, and how they affect our analysis. Two points are important. First, the locations of spiral arms systematically shift in the $v_{l}$ direction in an LVD due to non-circular motions, which cause errors in $R$ in our analysis (Section 3.2). Second, even with increased velocity widths due to enhanced velocity dispersions and spiral arm streaming motions, interarm regions are still sampled in an LVD.

Figure 12(a) demonstrates the systematic shifts of spiral arm locations from the circular rotation model (dotted lines) to the non-circular motion model (solid). Spiral arms show loops in the LVD and tend to appear squashed in the velocity direction due to the non-circular motions. This squashing is primarily due to the systematic change of velocity vector directions with respect to the directions of our lines of sight. For example, if the northern side of the Scutum-Crux arm is considered (Figure 11(a); $l \sim 0^{\circ}-30^{\circ}$ ), then the velocity vectors at its far side rotate away from our lines of sight due to the non-circular motions, while those at the near side rotate toward them. This shows that the squashed spiral loops are a general consequence of spiral arm non-circular motions in the MW.

The shifts in arm locations in an LVD result in systematic shifts in $R$ when Equation (5) is used. Figure 12(a) also shows constant $R$ lines. Figures 12(c) and (d) qualitatively demonstrate how these shifts affect radial profiles, separately for the northern and southern sides of the MW disk. The arrows indicate the directions and (very roughly) amounts of systematic shifts in $R$. If an underlying radial profile of, e.g., $f_{\text {mol }}$, follows the black solid line, then the profile would shift in the directions of the arrows. As a result, it would appear 

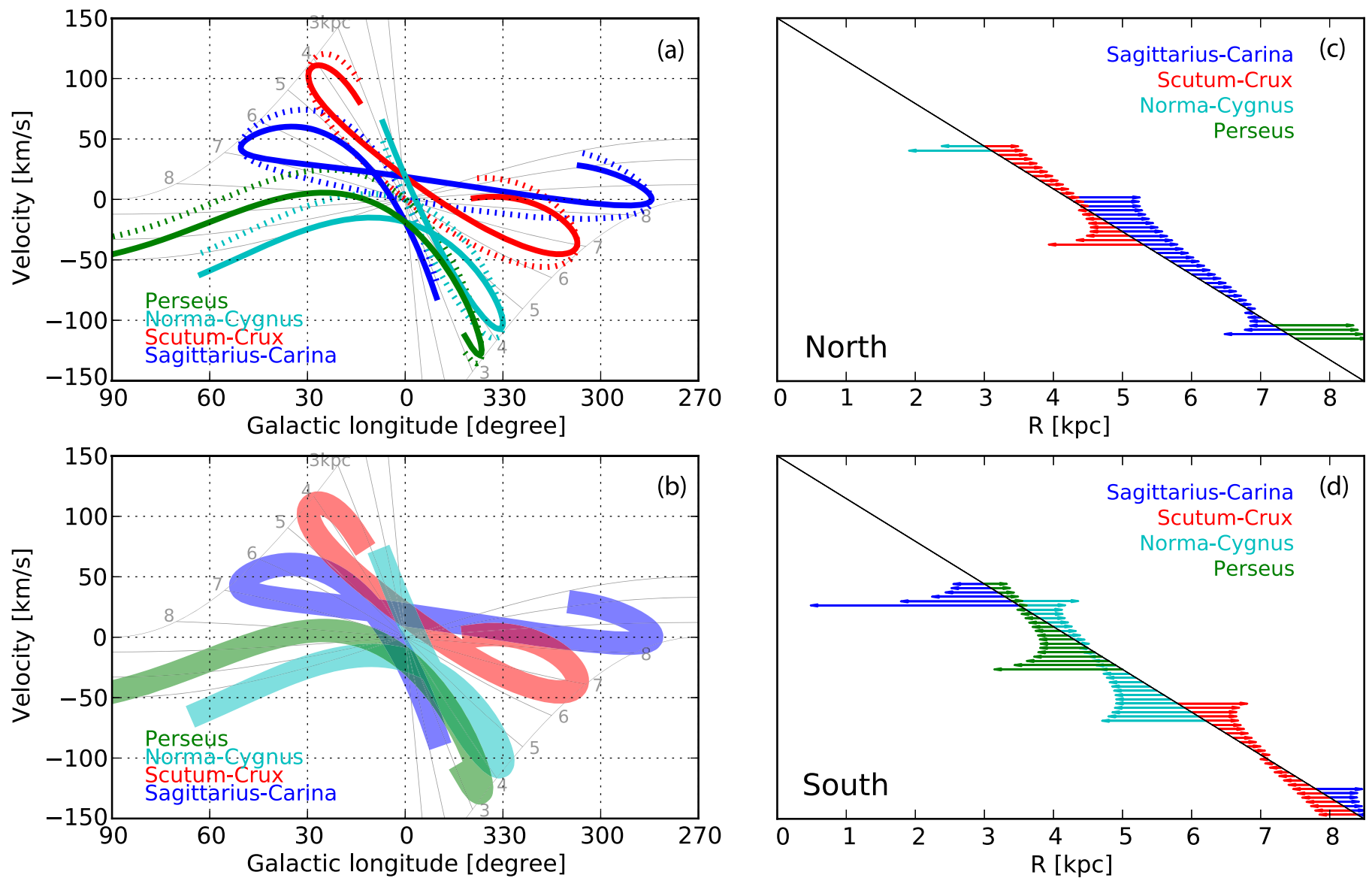

Figure 12. Model $l-v$ diagrams. (a) The same as Figure 11(b). Four spiral arms are plotted in the cases of pure circular rotation (dotted lines) and with non-circular motions (solid lines). (b) Four spiral arms with non-circular motions. The widths of the lines correspond to $20 \mathrm{~km} \mathrm{~s}^{-1}$ (arbitrarily chosen) to demonstrate the effect of velocity dispersions and streaming motions in spiral arms. The actual widths are likely smaller by a factor of about 2, especially for the Sagittarius-Carina and NormaCygnus arms that have no enhancement in stellar density (potential). (c), (d) Systematic errors in $R$ at positions along spiral arms due to non-circular motions: (c) for the northern, $0^{\circ}<l<90^{\circ}$ and $v>0 \mathrm{~km} \mathrm{~s}^{-1}$, and (d) southern disk, $270^{\circ}<l<360^{\circ}$ and $v<0 \mathrm{~km} \mathrm{~s}^{-1}$. The arrows indicate the amounts of shifts from the true radii, i.e., the origins of the arrows. For example, if an underlying radial profile follows the black solid line, the profile would be steepened locally due to the non-circular motions (by connecting the arrowheads). For clarity, we applied Equation (8) and removed a couple of outermost arrows from the southern parts of Norma-Cygnus and Perseus arms which are the points around $(l, v) \sim(0,0)$ in panels (a) and (b).

steepened (locally in some $R$ range) due to non-circular motions. The degree of steepening, of course, depends on that of non-circular motions.

The effect of increased velocity widths is demonstrated in Figure 12(b). The width is manually set for the non-circular rotation model; it corresponds to $\sim 20 \mathrm{~km} \mathrm{~s}^{-1}$ when an arm runs horizontally in the LVD, and is even wider, up to $\sim 50 \mathrm{~km} \mathrm{~s}^{-1}$, around the tangent points of the spiral arms. Even such widened spiral arms do not completely fill the LVD. Therefore, the LVD samples the spiral arm and interarm regions, even though their true locations remain uncertain. The actual widths of the spiral arms are likely about twice as narrow in the velocity domain, and this figure likely shows their largest possible impact on the LVD. In fact, most observations of the spiral arm streaming motions and velocity dispersions in the MW indicate a smaller full width $\left(2-12 \mathrm{~km} \mathrm{~s}^{-1}\right.$; e.g., Clemens 1985; Alvarez et al. 1990; Oka et al. 2007). In addition, the Sagittarius-Carina and Norma-Cygnus arms do not show corresponding stellar spiral arm potentials (Drimmel \& Spergel 2001; Benjamin 2008; Robitaille et al. 2012), and the steaming motions are perhaps smaller around these arms. Spiral arm velocity widths appear to be $<20 \mathrm{~km} \mathrm{~s}^{-1}$ in numerical simulations of MW gas dynamics (Wada et al. 1994; Fux 1999; Bissantz et al. 2003; Pettitt et al. 2015).

\section{REFERENCES}

Alvarez, H., May, J., \& Bronfman, L. 1990, ApJ, 348, 495

Arnal, E. M., Bajaja, E., Larrarte, J. J., Morras, R., \& Pöppel, W. G. L. 2000, A\&AS, 142, 35

Bajaja, E., Arnal, E. M., Larrarte, J. J., et al. 2005, A\&A, 440, 767

Benjamin, R. A. 2008, in ASP Conf. Ser. 387, Massive Star Formation: Observations Confront Theory, ed. H. Beuther, H. Linz, \& T. Henning (San Francisco, CA: ASP), 375

Benjamin, R. A., Churchwell, E., Babler, B. L., et al. 2005, ApJL, 630, L149 Bigiel, F., Leroy, A., Walter, F., et al. 2008, AJ, 136, 2846

Binney, J., \& Merrifield, M. 1998, Galactic Astronomy (Princeton, NJ: Princeton Univ. Press)

Bissantz, N., Englmaier, P., \& Gerhard, O. 2003, MNRAS, 340, 949

Blitz, L., \& Rosolowsky, E. 2004, ApJL, 612, L29

Blitz, L., \& Rosolowsky, E. 2006, ApJ, 650, 933

Blitz, L., \& Shu, F. H. 1980, ApJ, 238, 148

Bolatto, A. D., Wolfire, M., \& Leroy, A. K. 2013, ARA\&A, 51, 207

Bronfman, L., Cohen, R. S., Alvarez, H., May, J., \& Thaddeus, P. 1988, ApJ, 324,248

Burton, W. B., Deul, E. R., \& Liszt, H. S. 1992, Saas-Fee Advanced Course 21: The Galactic Interstellar Medium, ed. W. B. Burton, B. G. Elmegreen, \& R. Genzel (Berlin: Springer)

Burton, W. B., \& Gordon, M. A. 1978, A\&A, 63, 7 
Chakrabarti, S., Laughlin, G., \& Shu, F. H. 2003, ApJ, 596, 220

Churchwell, E., Babler, B. L., Meade, M. R., et al. 2009, PASP, 121, 213

Clemens, D. P. 1985, ApJ, 295, 422

Cohen, R. S., Cong, H., Dame, T. M., \& Thaddeus, P. 1980, ApJL, 239, L53

Colombo, D., Hughes, A., Schinnerer, E., et al. 2014, ApJ, 784, 3

Crutcher, R. M. 2012, ARA\&A, 50, 29

Dame, T. M., Hartmann, D., \& Thaddeus, P. 2001, ApJ, 547, 792

Dame, T. M., Ungerechts, H., Cohen, R. S., et al. 1987, ApJ, 322, 706

Dickey, J. M., \& Lockman, F. J. 1990, ARA\&A, 28, 215

Dobbs, C. L., \& Bonnell, I. A. 2006, MNRAS, 367, 873

Dobbs, C. L., Bonnell, I. A., \& Pringle, J. E. 2006, MNRAS, 371, 1663

Drimmel, R. 2000, A\&A, 358, L13

Drimmel, R., \& Spergel, D. N. 2001, ApJ, 556, 181

Elmegreen, B. G. 1989, ApJ, 338, 178

Elmegreen, B. G. 1993, ApJ, 411, 170

Engargiola, G., Plambeck, R. L., Rosolowsky, E., \& Blitz, L. 2003, ApJS, 149,343

Field, G. B., Blackman, E. G., \& Keto, E. R. 2011, MNRAS, 416, 710

Francis, C., \& Anderson, E. 2012, MNRAS, 422, 1283

Fukui, Y., Kawamura, A., Wong, T., et al. 2009, ApJ, 705, 144

Fukui, Y., Okamoto, R., Kaji, R., et al. 2014, ApJ, 796, 59

Fukui, Y., Torii, K., Onishi, T., et al. 2015, ApJ, 798, 6

Fux, R. 1999, A\&A, 345, 787

Georgelin, Y. M., \& Georgelin, Y. P. 1976, A\&A, 49, 57

Grenier, I. A., Casandjian, J.-M., \& Terrier, R. 2005, Sci, 307, 1292

Hartmann, D., \& Burton, W. B. 1997, Atlas of Galactic Neutral Hydrogen (Cambridge: Cambridge Univ. Press)

Hartmann, L., Ballesteros-Paredes, J., \& Bergin, E. A. 2001, ApJ, 562, 852

Heyer, M., \& Dame, T. M. 2015, ARA\&A, 53, 583

Heyer, M. H., Brunt, C., Snell, R. L., et al. 1998, ApJS, 115, 241

Heyer, M. H., Corbelli, E., Schneider, S. E., \& Young, J. S. 2004, ApJ, 602,723

Heyer, M. H., \& Terebey, S. 1998, ApJ, 502, 265

Honma, M., Sofue, Y., \& Arimoto, N. 1995, A\&A, 304, 1

Hou, L. G., \& Han, J. L. 2014, A\&A, 569, A125

Hughes, A., Meidt, S. E., Colombo, D., et al. 2013, ApJ, 779, 46

Jackson, J. M., Rathborne, J. M., Shah, R. Y., et al. 2006, ApJS, 163, 145

Kalberla, P. M. W., Burton, W. B., Hartmann, D., et al. 2005, A\&A, 440, 775

Kalberla, P. M. W., \& Dedes, L. 2008, A\&A, 487, 951

Kalberla, P. M. W., Dedes, L., Kerp, J., \& Haud, U. 2007, A\&A, 469, 511

Kalberla, P. M. W., \& Kerp, J. 2009, ARA\&A, 47, 27

Kawamura, A., Mizuno, Y., Minamidani, T., et al. 2009, ApJS, 184, 1

Kellermann, K. I., \& Verschuur, G. L. 1988, Galactic and Extragalactic Radio Astronomy (2nd ed.; Berlin: Springer)

Kim, W.-T., \& Ostriker, E. C. 2002, ApJ, 570, 132

Koda, J. 2013, in ASP Conf. Ser. 476, New Trends in Radio Astronomy in the ALMA Era: The 30th Anniversary of Nobeyama Radio Observatory, ed. R. Kawabe, N. Kuno, \& S. Yamamoto (San Francisco, CA: ASP), 49

Koda, J., Sawada, T., Hasegawa, T., \& Scoville, N. Z. 2006, ApJ, 638, 191

Koda, J., Sawada, T., Wright, M. C. H., et al. 2011, ApJS, 193, 19

Koda, J., Scoville, N., Sawada, T., et al. 2009, ApJL, 700, L132

Koda, J., \& Sofue, Y. 2006, PASJ, 58, 299

Kwan, J., \& Valdes, F. 1987, ApJ, 315, 92

Malhotra, S. 1995, ApJ, 448, 138

Martos, M., Hernandez, X., Yáñez, M., Moreno, E., \& Pichardo, B. 2004, MNRAS, 350, L47

Miura, R. E., Kohno, K., Tosaki, T., et al. 2012, ApJ, 761, 37

Nakanishi, H., \& Sofue, Y. 2003, PASJ, 55, 191
Nakanishi, H., \& Sofue, Y. 2006, PASJ, 58, 847

Nakanishi, H., \& Sofue, Y. 2016, PASJ, 68, 5

Oka, T., Nagai, M., Kamegai, K., Tanaka, K., \& Kuboi, N. 2007, PASJ, 59, 15

Onodera, S., Koda, J., Sofue, Y., \& Kohno, K. 2004, PASJ, 56, 439

Oort, J. H., Kerr, F. J., \& Westerhout, G. 1958, MNRAS, 118, 379

Ostriker, E. C., McKee, C. F., \& Leroy, A. K. 2010, ApJ, 721, 975

Paladini, R., Davies, R. D., \& De Zotti, G. 2004, MNRAS, 347, 237

Pettitt, A. R., Dobbs, C. L., Acreman, D. M., \& Bate, M. R. 2015, MNRAS, 449, 3911

Pety, J., Schinnerer, E., Leroy, A. K., et al. 2013, ApJ, 779, 43

Pineda, J. L., Langer, W. D., Velusamy, T., \& Goldsmith, P. F. 2013, A\&A, 554, A103

Planck Collaboration, Ade, P. A. R., Aghanim, N., et al. 2011, A\&A, 536, A19

Rand, R. J., \& Kulkarni, S. R. 1990, ApJL, 349, L43

Robin, A. C., Marshall, D. J., Schultheis, M., \& Reylé, C. 2012, A\&A, 538, A106

Robitaille, T. P., Churchwell, E., Benjamin, R. A., et al. 2012, A\&A, 545, A39

Roman-Duval, J., Jackson, J. M., Heyer, M., et al. 2009, ApJ, 699, 1153

Roman-Duval, J., Jackson, J. M., Heyer, M., Rathborne, J., \& Simon, R. 2010 , ApJ, 723, 492

Sanders, D. B., Scoville, N. Z., \& Solomon, P. M. 1985, ApJ, 289, 373

Sanders, D. B., Solomon, P. M., \& Scoville, N. Z. 1984, ApJ, 276, 182

Schinnerer, E., Meidt, S. E., Pety, J., et al. 2013, ApJ, 779, 42

Scoville, N. Z., \& Hersh, K. 1979, ApJ, 229, 578

Scoville, N. Z., \& Sanders, D. B. 1987, Interstellar Processes, Vol. 134, ed. D. J. Hollenbach, \& H. A. Thronson, Jr. (Netherlands: Springer), 21

Scoville, N. Z., \& Wilson, C. D. 2004, in ASP Conf. Ser. 322, The Formation and Evolution of Massive Young Star Clusters, ed. H. J. G. L. M. Lamers, L. J. Smith, \& A. Nota (San Francisco, CA: ASP), 245

Scoville, N. Z., Yun, M. S., Sanders, D. B., Clemens, D. P., \& Waller, W. H. 1987, ApJS, 63, 821

Sheth, K., Vogel, S. N., Regan, M. W., et al. 2002, AJ, 124, 2581

Sofue, Y., Honma, M., \& Arimoto, N. 1995, A\&A, 296, 33

Solomon, P. M., \& Edmunds, M. G. 1980, in Proc. Third Gregynog Astrophysics Workshop, University of Wales, Cardiff, Wales, August 1977 (Oxford and New York: Pergamon), 356

Solomon, P. M., Rivolo, A. R., Barrett, J., \& Yahil, A. 1987, ApJ, 319, 730

Steiman-Cameron, T. Y., Wolfire, M., \& Hollenbach, D. 2010, ApJ, 722,1460

Tachihara, K., Toyoda, S., Onishi, T., et al. 2001, PASJ, 53, 1081

Tan, J. C. 2000, ApJ, 536, 173

Tasker, E. J., \& Tan, J. C. 2009, ApJ, 700, 358

Tosaki, T., Kuno, N., Onodera, R. S. M., et al. 2011, PASJ, 63, 1171

Vallée, J. P. 2014, ApJS, 215, 1

Vallée, J. P. 2016, AJ, 151, 55

van Dishoeck, E. F., \& Black, J. H. 1988, ApJ, 334, 771

Vogel, S. N., Kulkarni, S. R., \& Scoville, N. Z. 1988, Natur, 334, 402

Wada, K. 2008, ApJ, 675, 188

Wada, K., \& Koda, J. 2004, MNRAS, 349, 270

Wada, K., Taniguchi, Y., Habe, A., \& Hasegawa, T. 1994, ApJL, 437, L123

Wegg, C., Gerhard, O., \& Portail, M. 2015, MNRAS, 450, 4050

Wolfire, M. G., Hollenbach, D., \& McKee, C. F. 2010, ApJ, 716, 1191

Wolfire, M. G., McKee, C. F., Hollenbach, D., \& Tielens, A. G. G. M. 2003, ApJ, 587, 278

Wong, T., \& Blitz, L. 2002, ApJ, 569, 157

Wyse, R. F. G. 1986, ApJL, 311, L41

Wyse, R. F. G., \& Silk, J. 1989, ApJ, 339, 700

Young, J. S., \& Scoville, N. Z. 1991, ARA\&A, 29, 581 